\title{
Une habitation et sa cave du premier âge du Fer en Centre Bretagne
}

A house and its cellar dating to the Early Iron Age in central Brittany

Sandrine Barbeau et Hélène Delnef

\section{(2) OpenEdition}

\section{Journals}

Édition électronique

URL : https://journals.openedition.org/rao/6253

DOI : $10.4000 /$ rao.6253

ISSN : $1775-3732$

Éditeur

Presses universitaires de Rennes

\section{Édition imprimée}

Date de publication : 18 décembre 2020

Pagination : $99-119$

ISBN : 978-2-7535-8232-3

ISSN : 0767-709X

Référence électronique

Sandrine Barbeau et Hélène Delnef, «Une habitation et sa cave du premier âge du Fer en Centre Bretagne », Revue archéologique de l'Ouest [En ligne], 36 | 2020, mis en ligne le 18 décembre 2020, consulté le 22 août 2022. URL : http://journals.openedition.org/rao/6253 ; DOI : https://doi.org/ $10.4000 /$ rao.6253 


\title{
Une habitation et sa cave du premier âge du Fer en Centre Bretagne
}

\author{
$A$ house and its cellar dating to the Early Iron Age in central Brittany
}

\author{
Sandrine Barbeau ${ }^{a}$, Hélène Delnef ${ }^{\text {b }}$
}

\begin{abstract}
Résumé : La fouille des vestiges archéologiques à Bel Air, au sud-ouest de la commune de Saint-Caradec (Côtes-d'Armor), a permis la découverte d'un habitat de la fin du premier âge du Fer. L’occupation, datée entre les vi et v ve av. n. è., se caractérise par un bâtiment d'habitation circulaire édifié sur tranchée de fondation comportant trois grandes phases de construction au même emplacement avec une attache structurelle commune liée à l'existence d'une longue cave de $15 \mathrm{~m}^{2}$. Le site de Bel Air montre ici une évolution originale du bâtiment circulaire qui s'illustre par l'usage de la tranchée de fondation et l'adjonction d'une annexe liée à l'ouverture du bâtiment sur le côté est. À cette typologie inhabituelle, s'ajoute la présence de la cave, formant ainsi un ensemble unique en Bretagne.
\end{abstract}

\begin{abstract}
The excavation of the Bel Air site locates to the South-west of Saint-Caradec, has revealed the remains of a Late Iron Age settlement. The site, which dates between 6 th and 5 th century $B C$, is characterised by circular ditches that comprise three main building phases. Each building has its own $15 \mathrm{~m}^{2}$ cellar. The ditched round houses of Bel Air bear witness to an undocumented development of their architecture by the adding of an annex on the south-west entrance. This unusual construction and the presence of a cellar form an ensemble hitherto unknown in Brittany..
\end{abstract}

Mots clés : habitat circulaire, cave, premier âge du Fer, Bretagne.

Keywords: round house, cellar, early Iron Age, Brittany.

Le projet d'agrandissement de la zone d'activité de Bel Air au sud-ouest de la commune de Saint-Caradec (Côtes-d'Armor) a été à l'origine de l'opération de fouille qui s'est déroulée en 2016 sur une surface de $5600 \mathrm{~m}^{2}$ (fig. 1 et 2). Outre l'étude des vestiges antiques perçus lors du diagnostic, elle a permis la découverte d'un habitat de la fin du premier âge du Fer. L'occupation, datée entre les $\mathrm{VI}^{\mathrm{e}}$ et $\mathrm{V}^{\mathrm{e}}$ s. av. n. è., se caractérise par des bâtiments d'habitation circulaires édifiés sur des tranchées de fondation. Trois grandes phases de construction se succèdent au même emplacement, toutes trois liées à l'existence d'une cave longue de $15 \mathrm{~m}^{2}$ (fig. 3 et 4). Aucune limite fossoyée ou palissadée n'a été mise en évidence autour de l'habitat lors de la fouille.
Le site archéologique est localisé sur une crête en pente douce délimitée au nord-est et au sud-ouest par le creusement de vallées appartenant au bassin versant de l'Oust. Un sommet culminant à $174 \mathrm{~m}$ NGF au nord-ouest du site constitue le point haut de la zone.

\section{LeS HABITATIONS}

Détectées à environ $0,30 \mathrm{~m}$ de profondeur, directement sous le niveau de labour, trois tranchées, qui se superposent avec un léger décalage pour chacune d'entre elles, dessinent le plan de trois bâtiments circulaires d'un diamètre d'environ 7 m (fig. 5).

a Archéologue, Inrap Bretagne (sandrine.barbeau@inrap.fr)

b Céramologue, Inrap Normandie (helene.delnef@inrap.fr) 
Bretagne

Côtes d'armor

Saint-Caradec

Bel Air

Section(s) et parcelle(s):

$\mathbf{X I}-\mathbf{n}^{\circ} 23 p$

Coord. Lambert93 CC48

$x: 1263700$

$y: 7237500$

$z: 172,50 \mathrm{~m} \mathrm{NGF}$
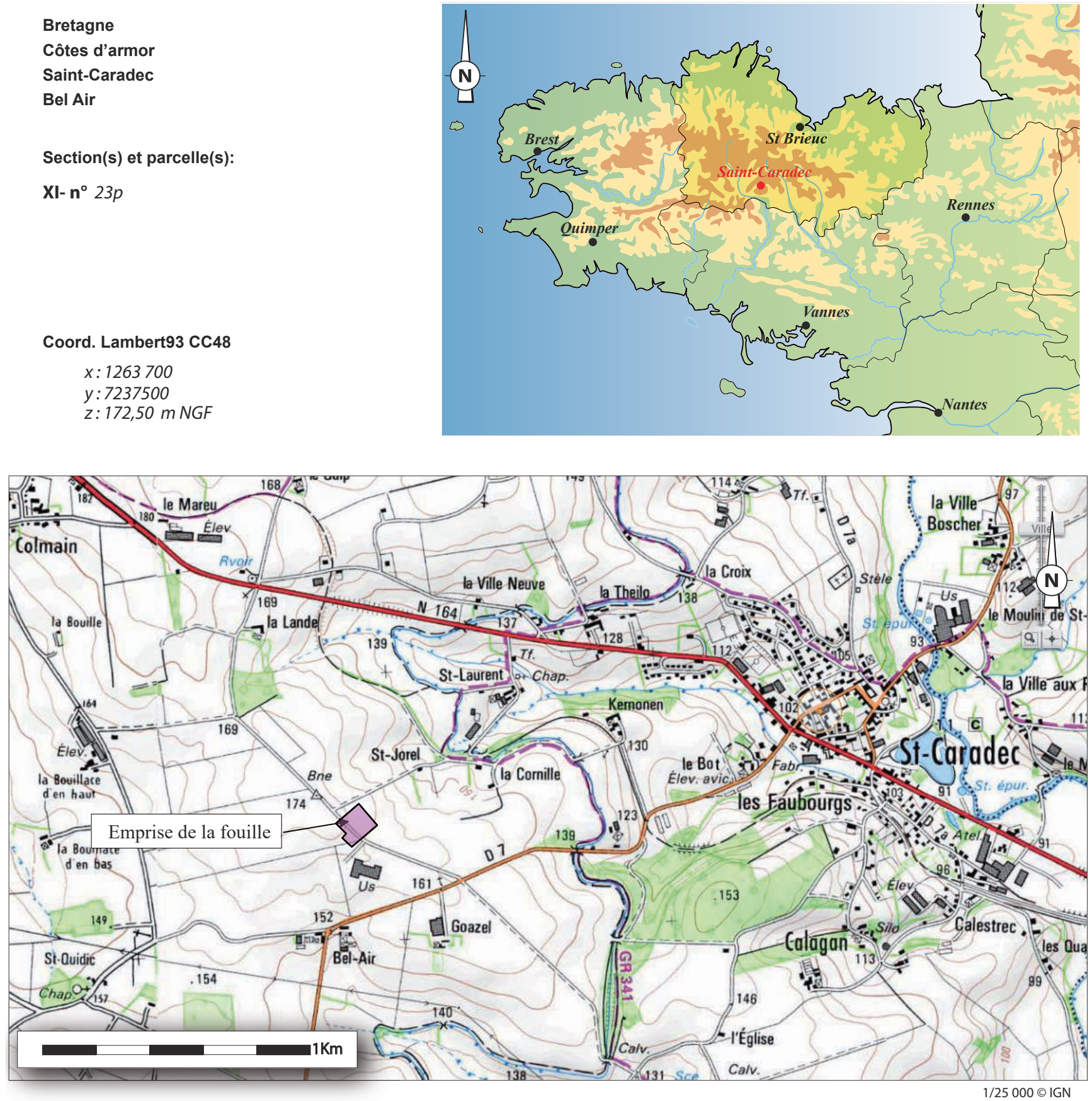

Figure 1 : Localisation du site de Saint-Caradec (Côtes-d'Armor) (DAO E. Collado, Inrap; source géoportail).

Figure 1: Location of Saint-Caradec (CAD E. Collado [Inrap]; source géoportail).

Une première grande phase de construction est représentée par les sections de tranchées 188, 189 et 53 qui forment un plan circulaire de 7,10 $\mathrm{m}$ de diamètre (fig. 6). Le bâtiment possède une ouverture à l'est, matérialisée par une interruption d'environ $1 \mathrm{~m}$ entre les tranchées et par l'emplacement de poteaux à chaque extrémité. De largeur irrégulière, le creusement de la tranchée 188 mesure entre
0,30 et $0,60 \mathrm{~m}$ de large pour une profondeur conservée de $0,06 \mathrm{~m}$ à $0,12 \mathrm{~m}$. Le profil, en cuvette large, est ponctué de creusements plus profonds correspondant à l'emplacement de poteaux qui s'assemblent parfois par paires. À son extrémité est, proches de l'entrée, des modifications structurelles sont perceptibles et signalent une reprise probable de l'édifice sur une petite portion. En effet, la tranchée 188 est 


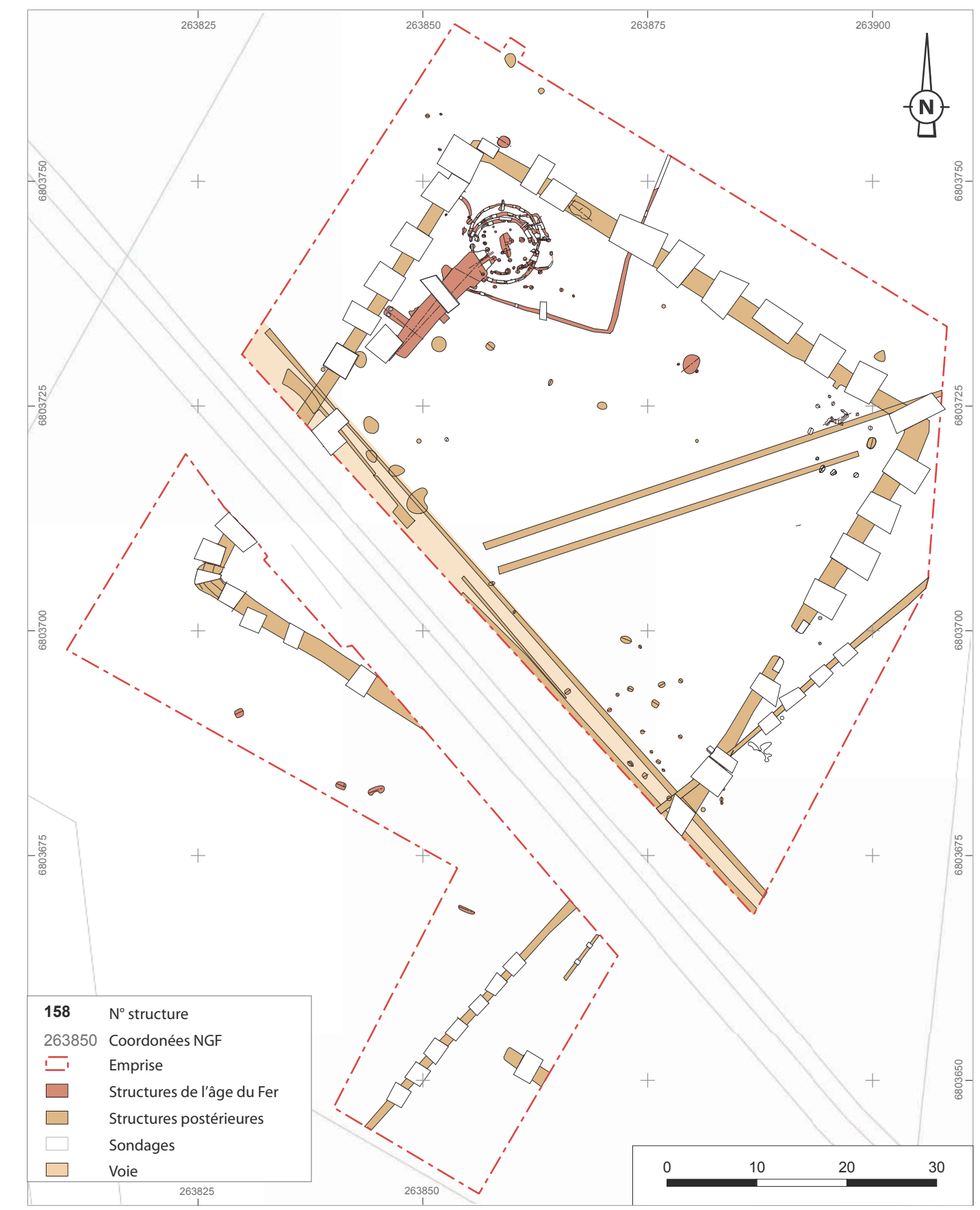

Figure 2 : Plan général des vestiges (DAO E. Collado, Inrap).

Figure 2: General plan of the site (CAD E. Collado, Inrap).

recoupée par le creusement de la tranchée 189 qui reprend et prolonge la première. Cette modification structurelle en plan s'accompagne d'un remplissage différencié qui révèle une reprise de paroi jusque dans sa fondation.

La tranchée 53 est la portion sud-est commune aux bâtiments des phases 1 et 2 . Cette tranchée apparaît, d'une manière générale, régulière, d'une largeur relativement constante de $0,30 \mathrm{~m}$ pour une profondeur conservée d'environ $0,14 \mathrm{~m}$ (fig. 6).
La partie sud-ouest du plan du bâtiment est occupée par le creusement de la cave (125) et une fosse d'installation de poteau (158), commune aux trois cercles d'habitation. Le trou de poteau 177, au centre de la construction, et les poteaux installés dans les tranchées participeraient au soutien de la charpente. Il n'a pas été possible de déterminer avec certitude si d'autres poteaux internes pouvaient être inclus dans ce plan en tant qu'éléments d'une structure porteuse. Néanmoins, la présence des poteaux proches de 


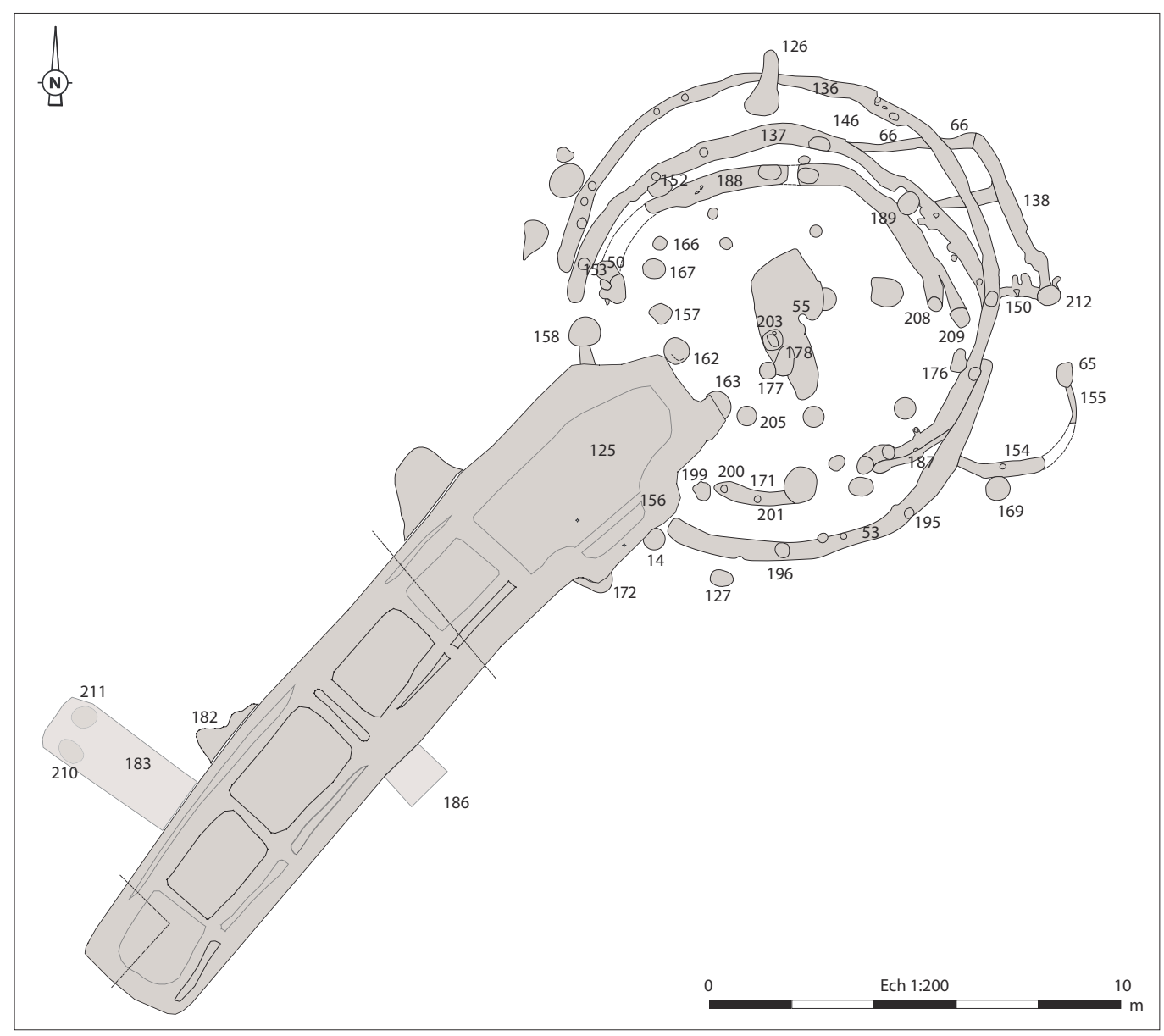

Figure 3 : Plan des différentes constructions et de la cave (DAO E. Collado, Inrap).

Figure 3: Plan of the different constructions and the cellar (CAD E. Collado, Inrap).

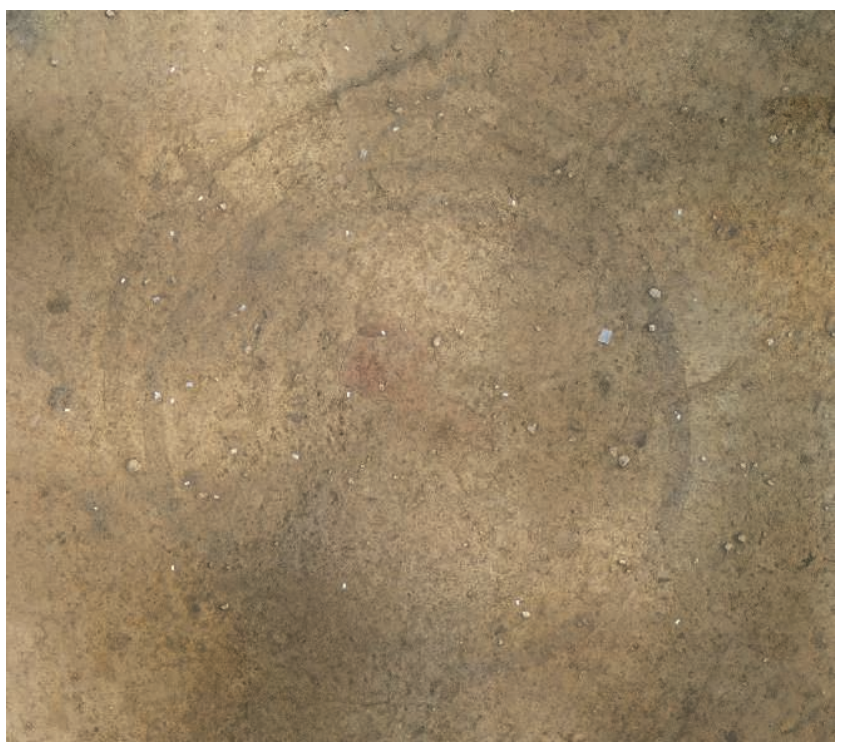

Figure 4 : Vue zénithale des bâtiments avant la fouille (cliché H. Paitier, Inrap).

Figure 4: Vertical view of the buildings before the excavation (photo H. Paitier, Inrap). l'entrée de la cave en tant que supplément dans le dispositif de charpente est également envisageable (fig. 3).

Le deuxième plan de construction, légèrement décalé vers le nord, comprend les tranchées 53 et 137. L'entrée reste, comme pour le premier plan de bâtiment, située à l'est avec un aménagement particulier associant les tranchées 66 et 138 (fig. 7).

La tranchée $137 \mathrm{a}$ une largeur de $0,20 \mathrm{~m}$ à $0,40 \mathrm{~m}$ pour une profondeur conservée en moyenne de $0,12 \mathrm{~m}$. Elle a un profil en $\mathrm{U}$ à fond plat, présentant régulièrement des creusements légèrement plus profonds qui signalent la présence de poteaux ou piquets. Le plan reste identique au premier avec en son centre le poteau 203 servant potentiellement de support à la charpente.

Pour ces deux premières phases, face aux poteaux d'entrée des constructions, les poteaux 65 et 212 (fig. 8) créent un ensemble qui rappelle le dispositif d'entrée sous porche à quatre poteaux tels que sur le site de la ZAC «Object'Ifs Sud " à Ifs (Calvados) (Besnard-Vautrin, 2011) ou celui de «La Verrie A 87 » (Vendée) (Pithon, 2000) (fig. 9). Cependant, les tranchées 138 et 154-155 auxquelles ces poteaux sont rat- 


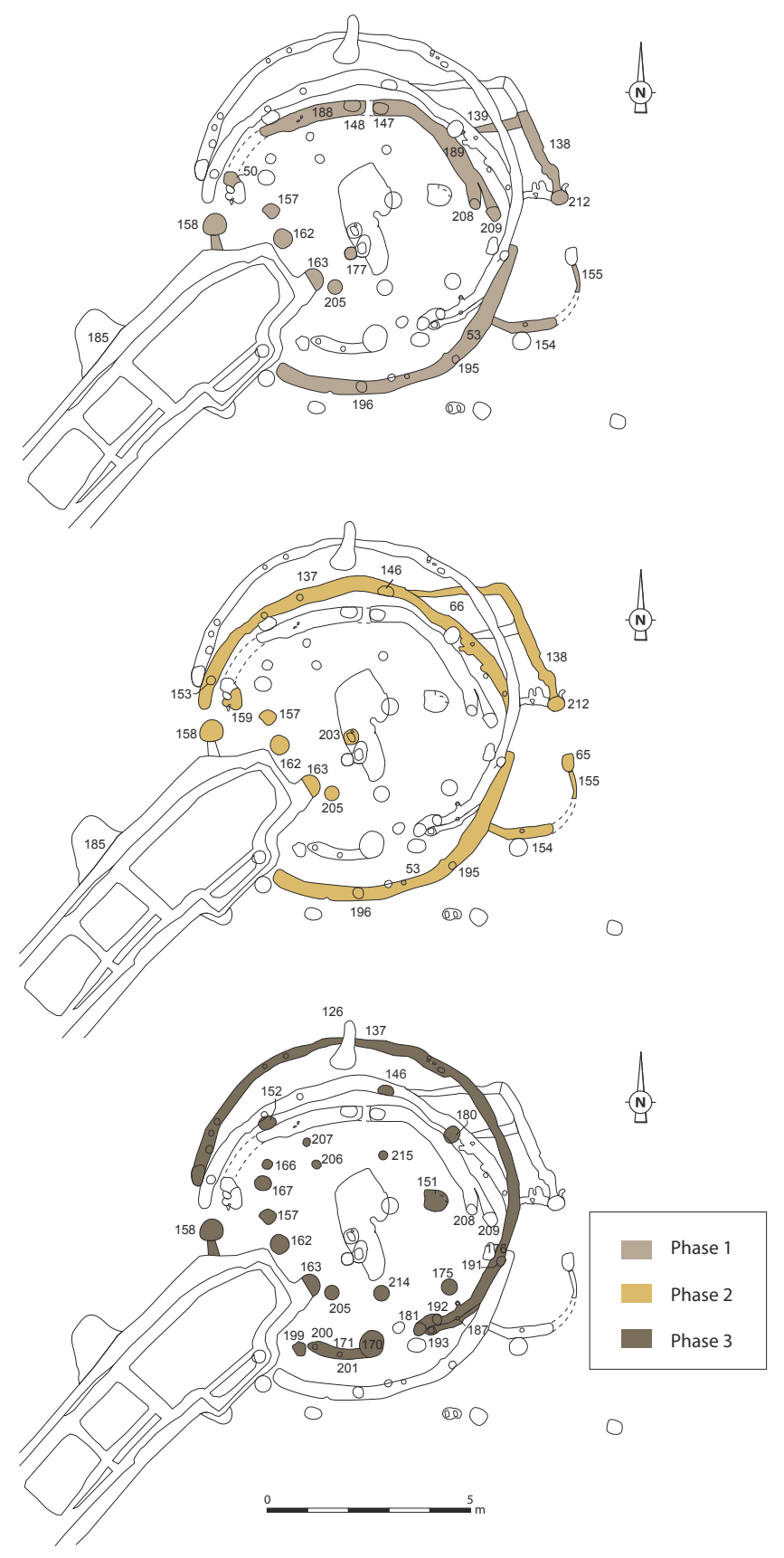

Figure 5 : Proposition des plans des différentes phases du bâtiment (DAO E. Collado, Inrap).

Figure 5: The different phases of the building (CAD E. Collado, Inrap).

tachés dessinent le plan d'un aménagement différent formant soit une cour fermée de mur, soit une annexe couverte. Cette proposition repose sur l'hypothèse d'une contemporanéité des structures, ce qui n'a cependant pas été démontré clairement. Ce plan de bâtiment comportant un appendice sur le côté est n'a pas d'équivalent sur les sites de cette période. Il évoque le plan de bâtiments fouillés en Angleterre sur le site de Butler's Field (Moore, 2003) ou encore celui de Shorncote (Moore, 2007) datés de la fin de l'âge du Fer ou encore, dans un contexte bien différent, ceux du site fortifié de Cividade de Terroso (Ayan Vila, 2008, p. 938) dans le nord du Portugal daté des $\mathrm{IV}^{\mathrm{e}}-\mathrm{II}^{\mathrm{e}} \mathrm{s}$. av. n. è. (fig. 10).

Le dernier édifice, de 7,30 $\mathrm{m}$ de diamètre, est matérialisé par les tranchées 136, 187 et 171 . Il est décalé vers le nord par rapport aux phases précédentes (fig. 5 et 11).

La tranchée 136 est large de $0,20 \mathrm{~m}$ à $0,30 \mathrm{~m}$ pour une profondeur maximale conservée de $0,24 \mathrm{~m}$. Ses parois sont droites à légèrement évasées et le fond étroit. Lors de la fouille intégrale de cette tranchée, différentes observations de son comblement et des impacts dans le fond amènent à penser que cette construction associait poteaux et piquets sur la section nord-est de la tranchée et utilisait des planches jointives sur la section ouest (fig. 12a et b). Cette différence interroge sur la possibilité d'une construction de paroi avec des techniques mixtes qui pourrait s'expliquer par le réemploi de matériaux issus des constructions précédentes.

Quant à la tranchée 187, large d'environ $0,40 \mathrm{~m}$ et peu profonde $(0,12 \mathrm{~m}$ maximum), elle a un profil aux parois verticales à légèrement évasées et un fond large relativement plat, mais assez irrégulier dans le creusement.

En complément de la tranchée 136, une couronne plus régulière de poteaux $(162,157,167,152,202,180,151$, 214, 205 et 163) ressort du plan d'ensemble (fig. 5). Ces derniers pourraient venir en support de la charpente avec l'aménagement d'une sablière haute sur laquelle reposeraient les entraits. Cette configuration permettrait éventuellement un support de plancher.

Ce type de plan avec une structure porteuse interne se rapproche de l'ensemble IV du site de Poses (Seine-Maritime) (Godard, 2013, p. 34) (fig. 9). Ce dernier, au plan incomplet d'environ $7 \mathrm{~m}$ de diamètre, possède 9 poteaux à l'intérieur dont l'un est central. À l'instar du plan du bâtiment de la phase 3, l'implantation des trous de poteau à Poses ne répond pas à un plan géométrique bien défini.

Le dernier plan de bâtiment comporte une entrée au sudest large d'environ 1,1 m, marquée par un poteau imposant au sud (170) et par deux poteaux côté sud-est $(181,190)$. Une deuxième entrée à l'ouest, où il existe également un espace entre l'extrémité sud de la tranchée 136 et le trou de poteau 158, est également envisageable.

Un tel arrangement se retrouve sur le site de L'Emonnière à La Verrie (Vendée) (Pithon, 2000) (fig. 9), où la construction circulaire comporte deux entrées dont une abritée sous un porche. Cette structure n'avait pu être datée avec précision mais appartenait plutôt à un contexte laténien. Lors de cette dernière phase, l'annexe à l'est ne serait pas conservée.

À l'intérieur des cercles d'habitation, plusieurs trous de poteau $(205,162,157,167)$ de fort diamètre apparaissent 

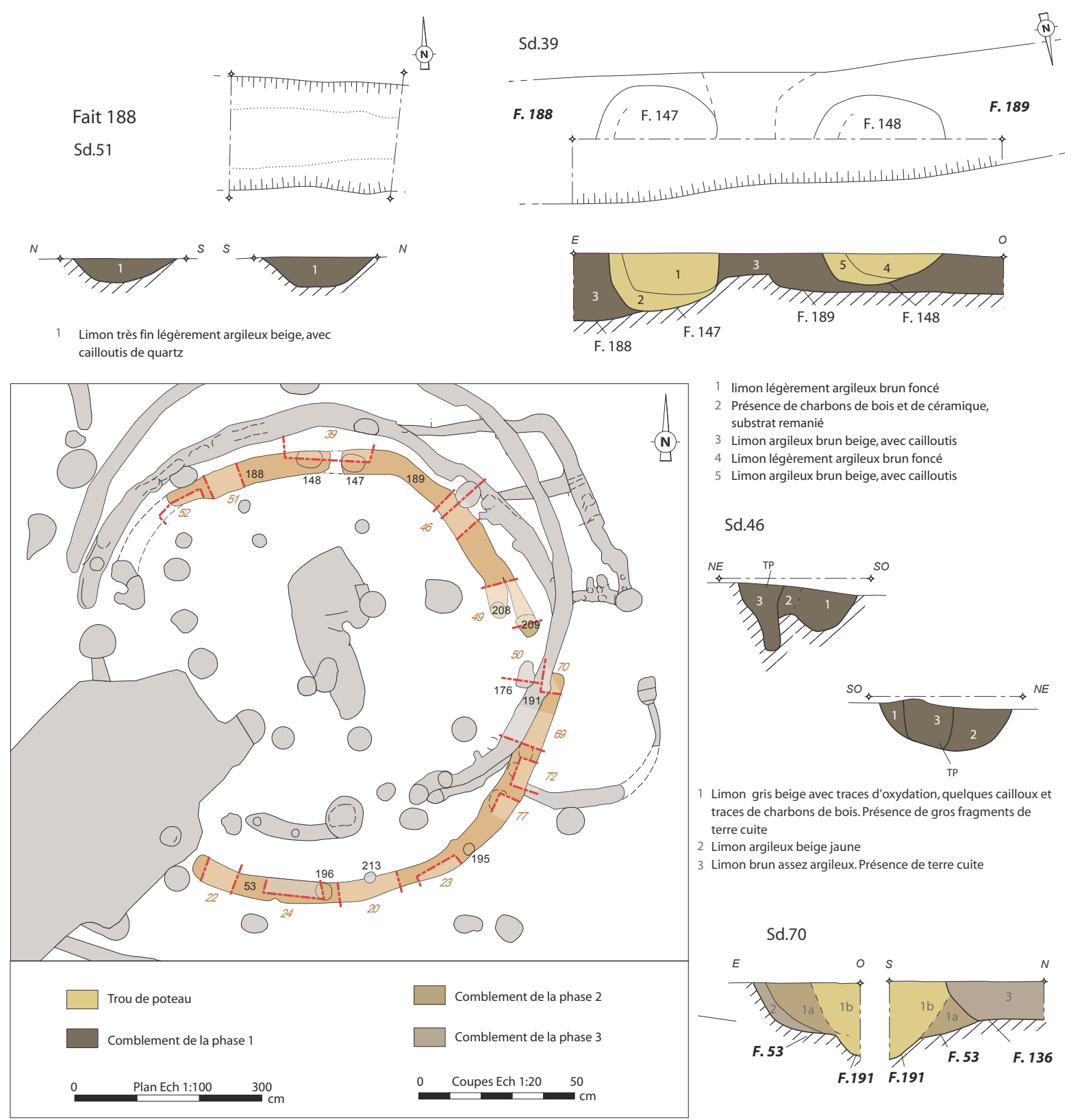

1 limon légèrement argileux brun foncé

2 Présence de charbons de bois et de céramique,

substrat remanié

3 Limon argileux brun beige, avec cailloutis

4 Limon légèrement argileux brun foncé

5 Limon argileux brun beige, avec cailloutis

Sd.46

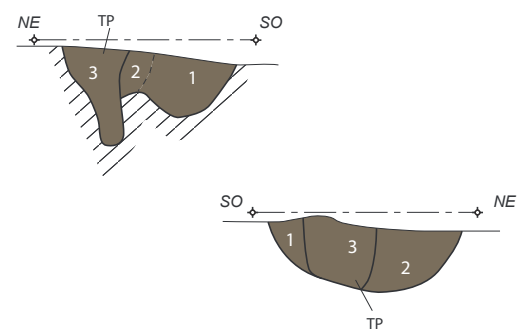

1 Limon gris beige avec traces d'oxydation, quelques cailloux et traces de charbons de bois. Présence de gros fragments de terre cuite

2 Limon argileux beige jaune

3 Limon brun assez argileux. Présence de terre cuite

Sd.70

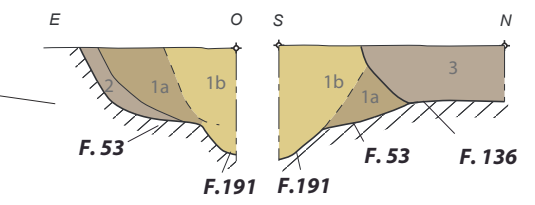

1a Limon argileux brun beige avec nodules de terre cuite et cailloutis. Présence de charbons de bois

1b Limon argileux brun moyen avec des boulette d'argile jaune et des charbons de bois

2 Limon brun moyen à gris avec quelques charbons de bois
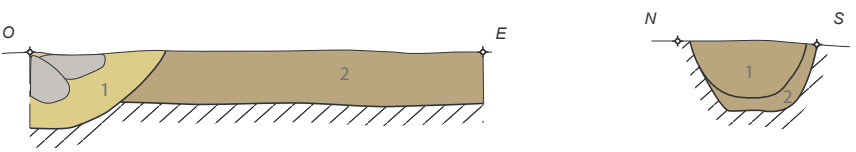

1 Limon brun avec charbons de bois

2 Limon brun, avec nombreuses inclusions de schiste. Présence de charbons de bois et de terre cuite Blocs de quartz

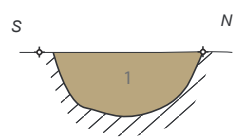

Limon argileux brun

nodules de schiste.

4 Présence de quelques charbons de bois

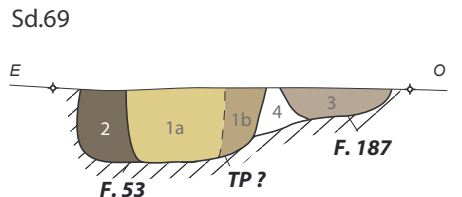

Figure 6: Coupe des tranchées 188, 189 et 53 (sd 51, 39, 46 et 20) (DAO E. Collado, Inrap).

Figure 6: Cuts of trenches 188, 189 and 53 (trenches 51,39, 46, and 20) (CAD E. Collado, Inrap). 
Sd.59
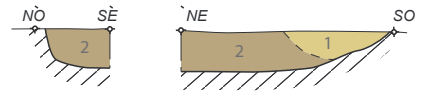

1 Limon brun gris avec charbons de bois

2 Limon beige
Sd. 55

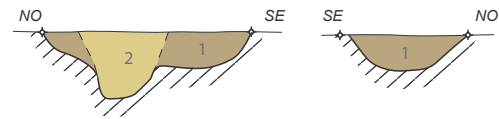

1 Limon brun gris

2 Limon argileux brun-gris avec charbons de bois

Sd.56
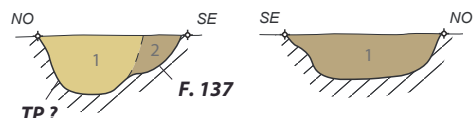

Limon brun gris avec charbons de bois

2 Limon argileux beige

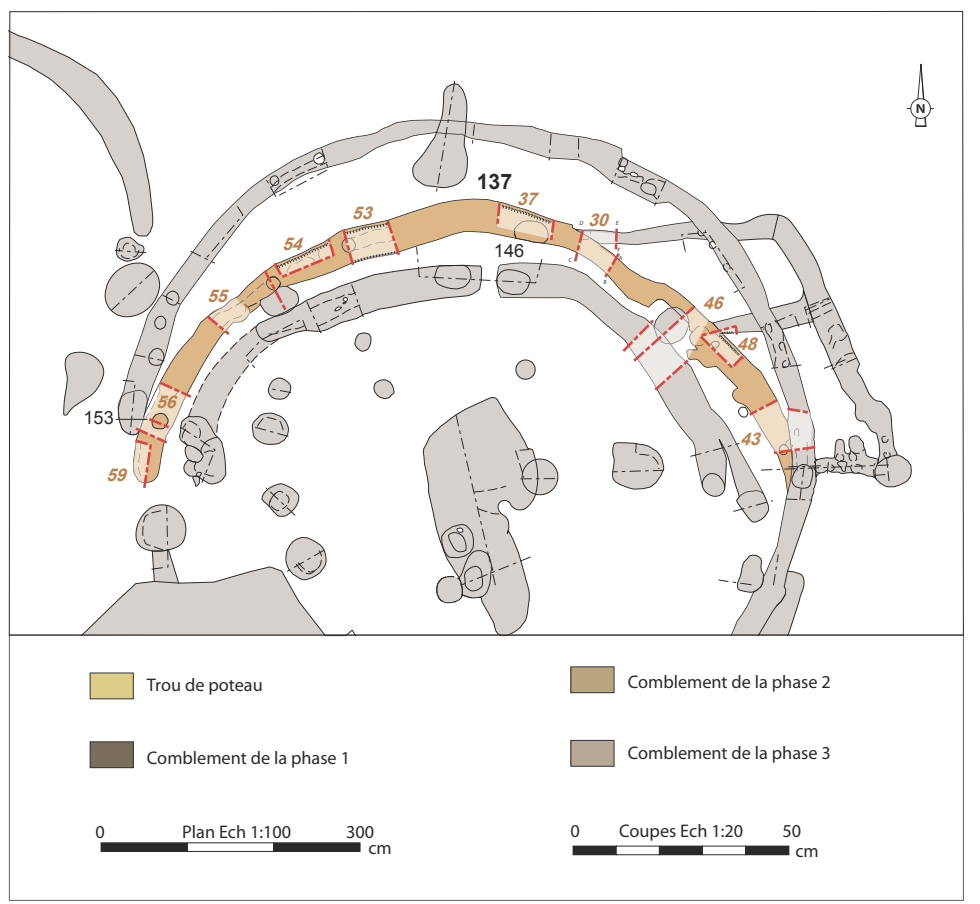

Sd. 48

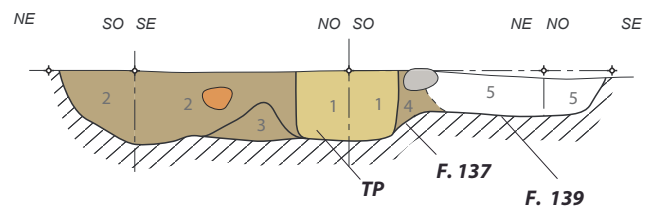

1 Limon assez argileux brun beige, avec traces d'oxydation au fond, quelques quartz, quelques traces de charbon de bois. Présence de terre cuite (poteau?)

2 Limon argileux beige, avec quelques cailloux de schiste et de quartz, traces de charbons de bois. Présence de terre cuite dans le comblement de tranchée avec du substrat

3 Limon argileux beige compact, avec quelques cailloux de schiste et de quartz, traces de charbons de bois. Présence de terre cuite

4 Limon assez argileux brun clair beige, avec traces d'oxydation au fond, quelques quartz, quelques traces de charbons de bois. Présence de terre cuite

5 Limon argileux beige, avec quelques cailloux de schiste et de quartz, traces de charbons de bois. Présence de terre cuite

Figure 7 : Coupes dans la tranchée 137 de la phase 2 (DAO E. Collado, Inrap). Figure 7: Cuts in trench 137 of phase 2 (CAD E. Collado, Inrap).
Sd.54

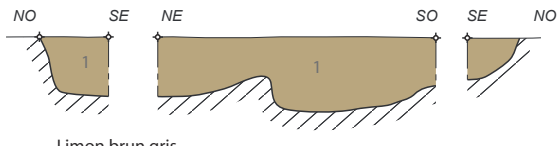

Limon brun gris

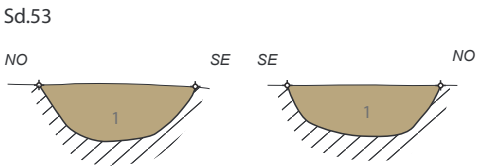

1 Limon très fin, argileux, très compact beige, avec cailloutis de quartz Sd.37

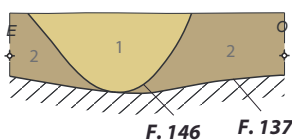

Limon légèrement argileux brun beige, meuble et

homogène. Rares charbons de bois. Présence de céramique

F. 146 F. 1372 Limon brun plutôt hétérogène avec lentilles de substrat remanié
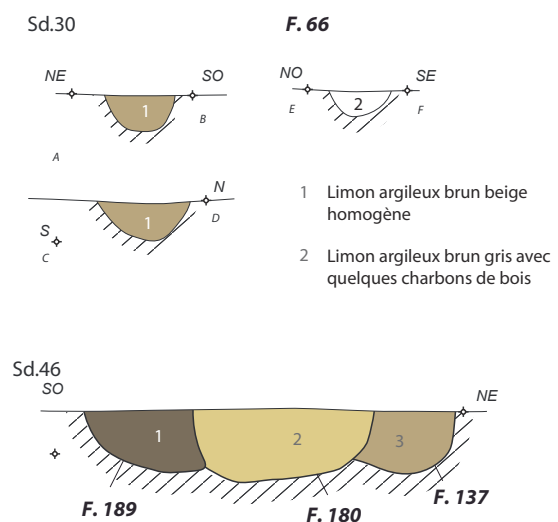

1 Limon brun assez argileux avec terre cuite en surface

2 Limon beige, avec quelques quartz et fragments de granite Présence de charbons de bois, de terre cuite et de céramique

3 Limon brun beige, avec quelques quartz

Sd. 43
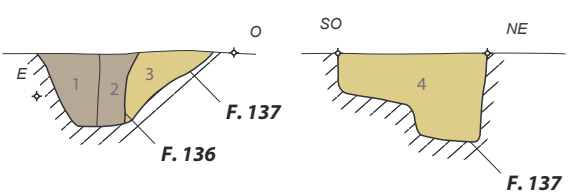
Limon argite

2 Limon argileux jaune (substrat remanié)

3 Limon argileux gris jaune. Présence de charbons de bois et de terre cuite

4 Limon argileux brun jaune. Présence de charbons de bois et de terre cuite rouge 


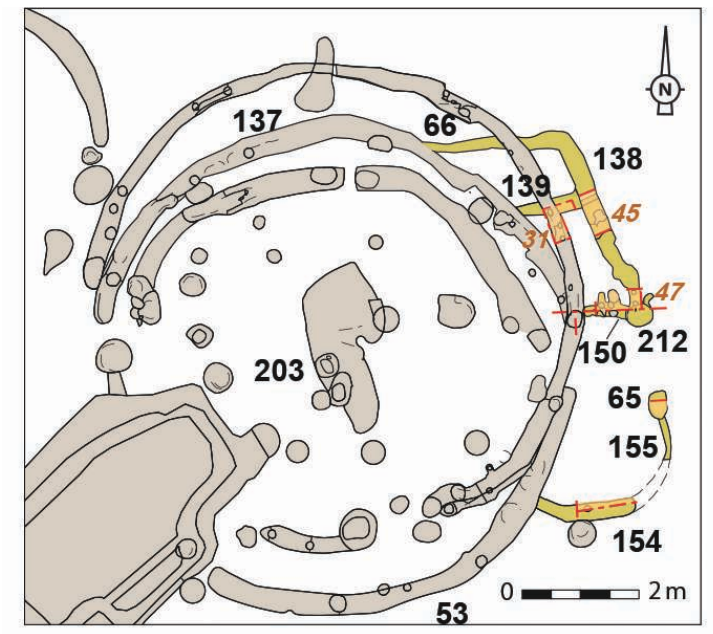

Faits 136-139 Sd.31

$\circ$

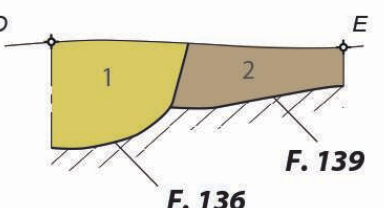

1 limon brun jaune avec inclusions de substrat argileux jaune clair et quelques pierres de quartz. Présence de charbons de bois et de terre cuite rouge

2 limon brun jaune grisâtre avec rares inclusions de charbons de bois

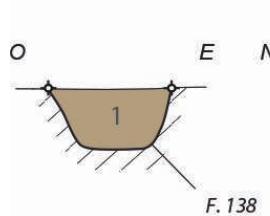

1 Limon argileux brun jaune, avec terre cuite

Sd.47

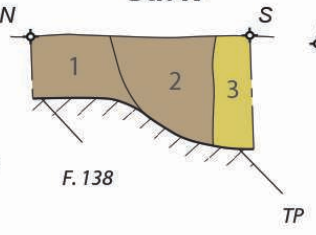

1 Limon argileux brun jaune, avec charbons de bois

2 Limon argileux brun gris, avec charbons de bois, céramique et nombreux fragments de terre cuite

3 L.imon argileux brun foncé, avec charbons de bois, céramique et nombreux fragments de terre cuite

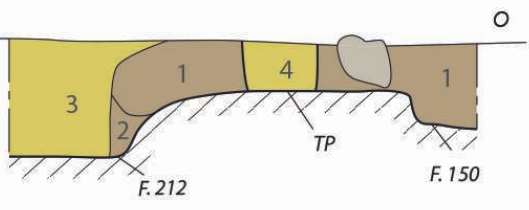

1 Limon argileux brun jaune

2 Limon argileux brun gris, avec charbons de bois et terre cuite rouge

3 Limon argileux brun foncé, avec charbons de bois

4 Limon argileux brun jaune grisâtre, avec charbons de bois et terre cuite

\section{Fait 138 Sd.45}

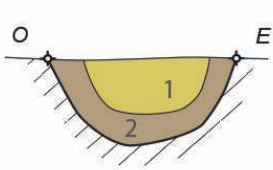

1 Limon argileux brun, avec charbons de bois. Présence de céramique et de nombreux fragments de terre cuite

2 Limon argileux jaune brun avec charbons de bois
Comblement

Trace du poteau

Ech 1:20
Figure 8 : Aménagement de l'entrée au nordest : une cour? (DAO E. Collado, Inrap). Figure 8: The north-east entrance showing a yard? (CAD E. Collado, Inrap). potentiellement fonctionnels dans les trois phases de construction. Ce type d'architecture circulaire sur tranchée de fondation ne nécessite pas obligatoirement de poteaux internes pour soutenir la toiture (Dechezleprêtre, 1997, p. 55-56). Or, ces poteaux à l'intérieur du bâtiment à Bel Air ont la particularité d'être localisés auprès de l'entrée de la cave. Leur installation est sans doute à mettre en relation avec l'existence de cette excavation et la nécessité d'une structure porteuse plus conséquente de ce côté, pour pallier le creusement large de près de $3 \mathrm{~m}$ à l'emplacement de la paroi du mur.

La structure de combustion 55 occupe une position centrale au sein de ces bâtiments. Elle dessine une zone rubéfiée de forme irrégulière de 2,60 $\mathrm{m}$ de long pour 1,30 $\mathrm{m}$ de large au maximum. Le foyer est composé de deux parties : l'une d'environ 1,20 m de diamètre, très rubéfiée en surface, à laquelle est accolée à l'est une autre zone moins rubéfiée et de forme irrégulière (sur $1 \mathrm{~m}$ de long environ). La fouille a mis en évidence, pour la première, un petit creusement en cuvette de $1 \mathrm{~m}$ de diamètre pour $0,16 \mathrm{~m}$ de profondeur. La deuxième partie pourrait correspondre au foyer des phases 1 et 2 de l'habitat (fig. 13).

\section{Étude du mobilier céramique des habitats (par Hélène Delnef)}

L'ensemble céramique découvert sur la totalité du site de Saint-Caradec comptabilise 422 tessons pour 36 individus au minimum.

Les récipients étudiés sont montés majoritairement à la main avec une cuisson réductrice en mode B et B' (Vaginay, 
Figure 9 : Comparaison des bâtiments circulaires de l'âge du Fer en Bretagne et en Normandie (DAO : E. Collado, Inrap, d'après Le Gall, 2017 ; Lefort, 2010 ; Pithon, 2000 ; Godard, 2013; Dechezleprêtre et al., 1997).

Figure 9: Comparison of circular Iron Age buildings in Brittany and Normandy (CAD E. Collado, Inrap, after Le Gall, 2017; Lefort, 2010; Pithon, 2000; Godard, 2013; Dechezleprêtre et al., 1997).
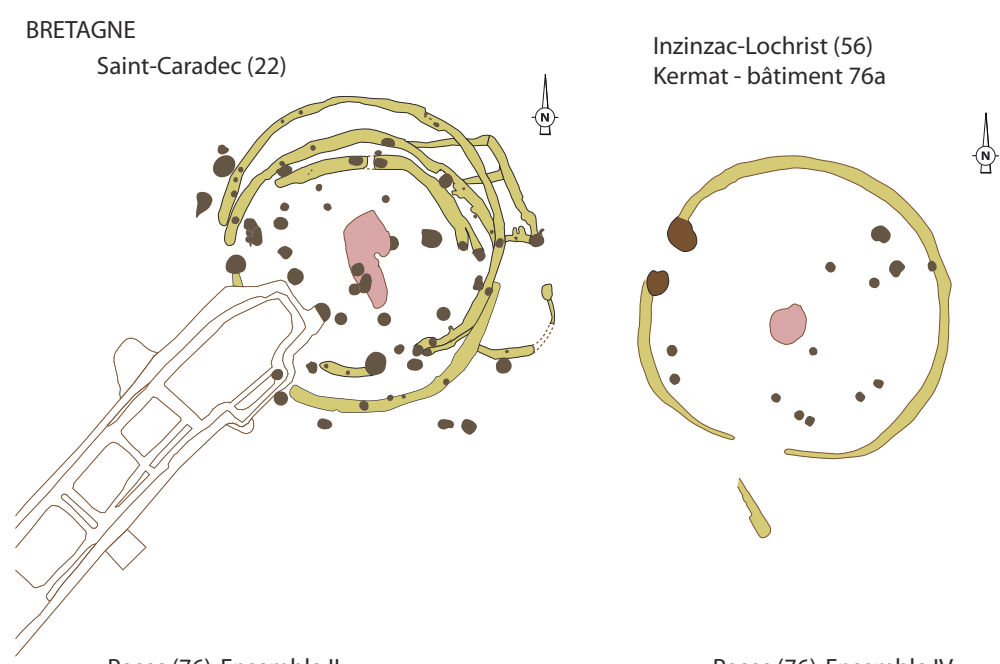

Poses (76), Ensemble II

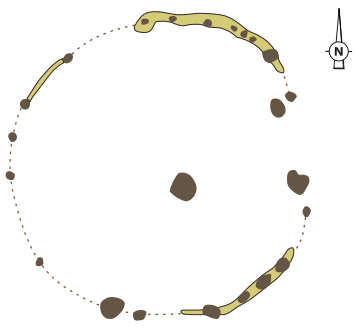

Poses (76), Ensemble IV

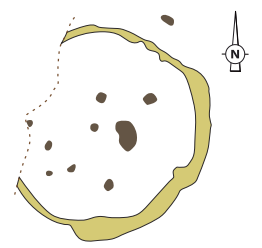

NORMANDIE

Urville-Nacqueville (50), structure 4

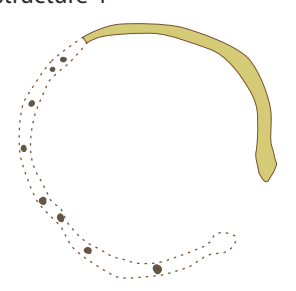

Restitution

Trous de poteaux
Tranchée d'implantation de la paroi

Structure de combustion
PAYS DE LA LOIRE

La Verrie (85) - A87
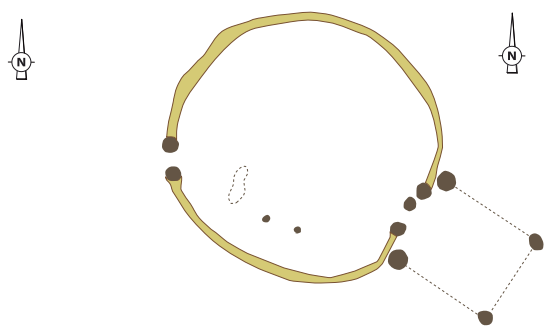

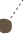

\section{.}

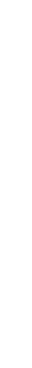


Shorncote1997-8 (Royaume-Uni), fin de l'âge du Fer
Larmor-plage, Quelisoye (Morbihan), La Tène Finale

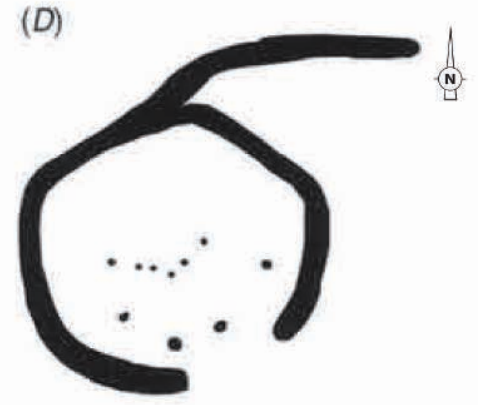

Buttler's Field (Royaume-Uni),

fin de l'âge du Fer

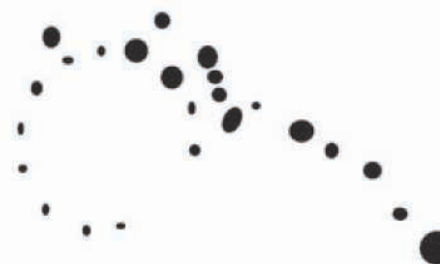

Ech $1: 400 \quad 10$

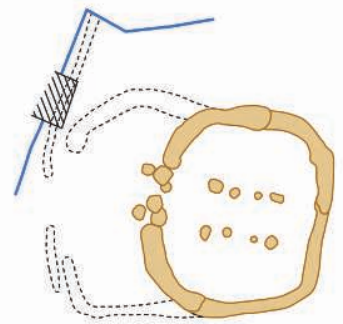

PORTUGAL

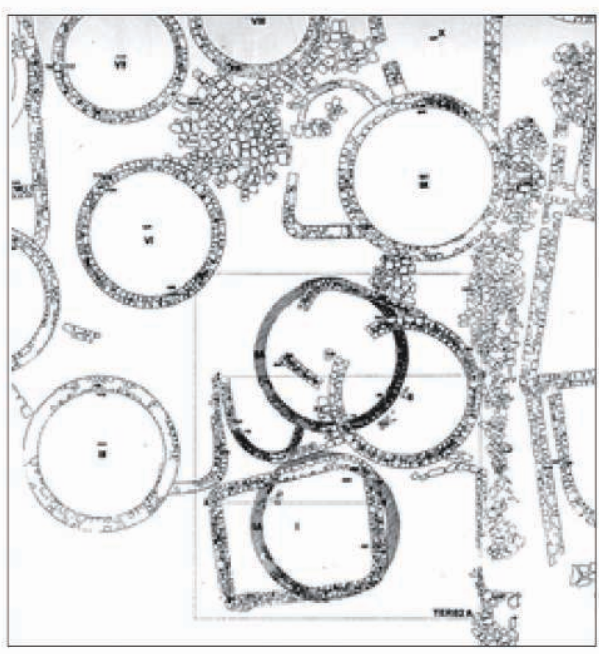

Plan des niveaux d'occupation du premier âge du Fer à Cividade de Terroso (Silva 1986:39-40 ; planche XVIII).
Figure 10 : Comparaison des plans des bâtiments circulaires de Bel Air avec des bâtiments de l'âge du Fer des sites de Bretagne, d'Angleterre et du nord du Portugal (DAO E. Collado, Inrap, d'après Blanchet, 2016; Moore, 2007; Moore, 2003 in Moore, 2007; Silva, 1986 in Ayàn Vila, 2008).

Figure 10: Comparison of the plans of circular buildings of Bel Air with Iron Age constructions in Brittany, Britain and northern Portugal (CAD E. Collado, Inrap, after Blanchet, 2016; Moore, 2007; Moore, 2003 in Moore, 2007; Silva, 1986 in Ayàn Vila, 2008).
Des comparaisons peuvent être trouvées dans des habitats de la deuxième moitié du $\mathrm{VI}^{\mathrm{e}} \mathrm{s}$. à la deuxième moitié du $\mathrm{IV}^{\mathrm{e}} \mathrm{s}$. (Cherel et al., 2018, type 18), comme à Plouër-sur-Rance dans la première phase ancienne datée de la première moitié du ve s. (Menez, 1996 : fig. 100/type 10) ou à Plérin, dans l'état 3 du fossé 2007 (sd 3020, us 2, Le Gall, 2013 : fig. 35) daté de la seconde moitié du v $v^{e} s$. au tout début du Iv $v^{e} s$.

Dans le trou de poteau 162, situé près de l'ouverture de la cave, deux formes sont individualisées. La première est une encolure évasée et la deuxième est un récipient caréné à large bord rectiligne, épaule et panse tronconique et carène haute. Ce type est présent dans l'état 1 du fossé 2007 de Plérin, rue de l'Arrivée, daté entre la fin du $\mathrm{vI}^{\mathrm{e}}$ et le début du v $\mathrm{v}^{\mathrm{e}} \mathrm{s}$. (Le Gall, 2013 : fig. 37).

Dans le fait 138 (sd 47), la présence d'un tesson à décor estampé est notable. Ce motif en " équerre perlée " est organisé en frise encadrée de filets incisés. Cette forme d'orne- mentation apparaît vers le début du $\mathrm{v}^{\mathrm{e}} \mathrm{s}$. (Cherel et al., $2018: 304)$.

Le fait 170 a livré un petit bord à lèvre amincie qui évoque par sa facture les productions de la fin de la période hallstattienne et du début de la période laténienne.

Au sein de l'ensemble architectural, des tessons ont été isolés lors de la fouille. Parmi ceux-ci, deux encolures à bord évasé et léger méplat interne sont recensées (UA 1, iso 10 et UA 1, iso 4 et 7). Leur profil est proche des récipients du fait 146 dans le sondage 37 (us 1) et du fait 163. Ce type de bord est également fréquent entre le Hallstatt D3 et le début de La Tène ancienne.

D'après le mobilier céramique étudié, les différentes phases de construction de ce bâtiment se sont enchaînées sur une période relativement courte, entre la fin du Hallstatt D3 et le début de La Tène ancienne. 


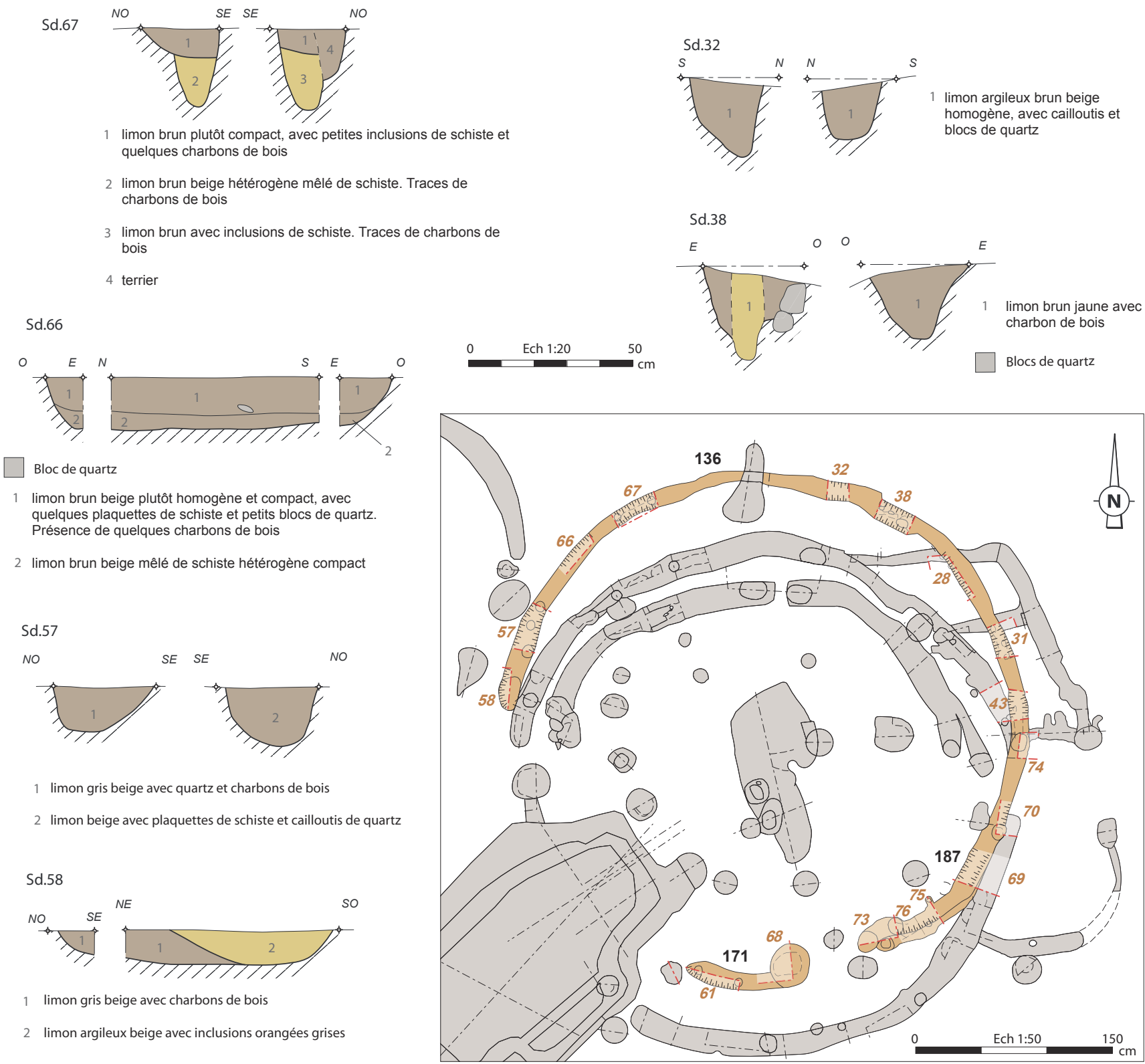

Figure 11 : Plan de la troisième phase de construction (136,187 et 171) et localisation des sondages. (DAO E. Collado, Inrap). Figure 11: Plan of the third building phase (136,187 and 171) and location of the trenches. (CAD E. Collado, Inrap).

La durée de vie de chacun des bâtiments peut être estimée entre 50 et 60 ans par maison si l'on considère les datations fournies par la céramique qui indiquent une période d'occupation de 150 ans à 200 ans au maximum. Les causes de ces reconstructions seraient davantage liées à la dégradation des matériaux ou peut-être des accidents liés au feu (phase 1) qu'à une volonté d'agrandissement, étant donné la faible augmentation de superficie habitable qui passe de 40 à $42 \mathrm{~m}^{2}$.

Les plus grands changements observés s'opèrent lors de la dernière grande phase de construction, qui garde néanmoins une partie de plan en commun avec les plans des constructions précédentes. Cette partie correspond à l'emplacement de la cave qui semble être la charnière structurelle et temporelle de ce secteur d'habitation. Elle apparait en tout cas comme étant un facteur essentiel de ces constructions successives.

La réalisation de la photographie verticale décorrélée a permis de mettre en évidence le tracé des tranchées passant sur le comblement de la cave (une lisibilité faible en plan et à la fouille) (fig. 15). Cette limite entre habitation et comblement de la cave correspond en outre à un changement dans le com- 


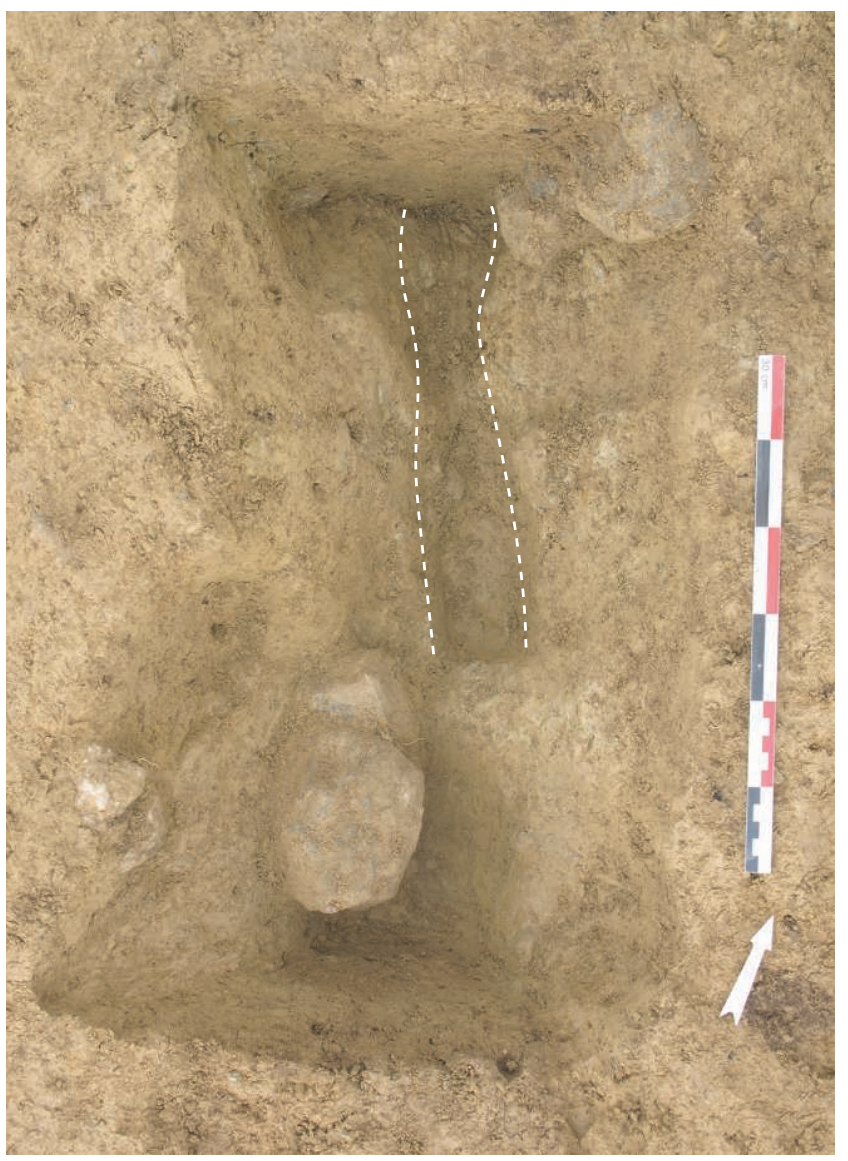

Figure 12a : Vue de la coupe de la tranchée 136 dans le sondage 38 (clichés V. Chaigne, Inrap).

Figure 12a: View of the cut of the foundation trench 136 in trench 38 (photos V. Chaigne, Inrap).

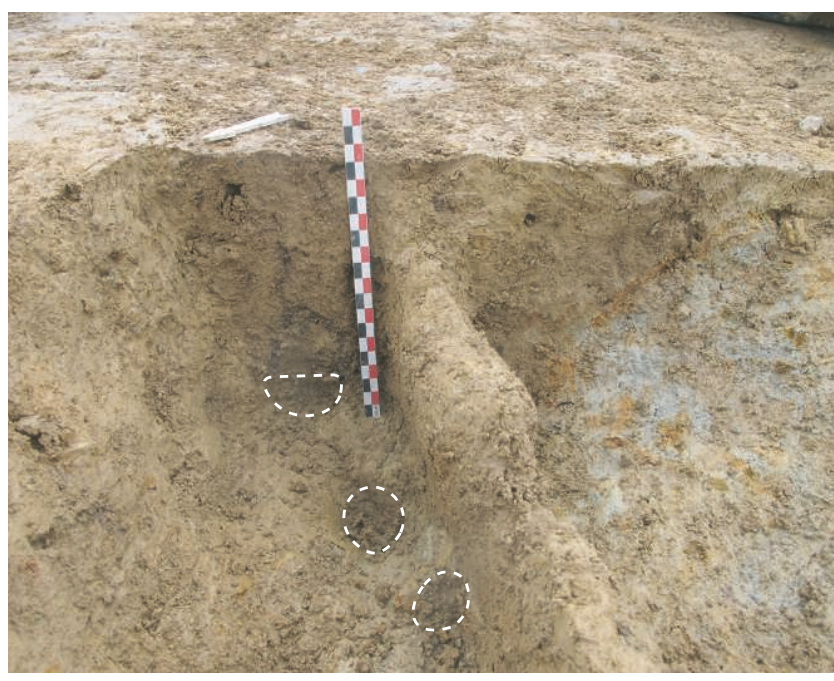

Figure 12b : Vue de la coupe de la tranchée 136 dans le sondage 43 (cliché S. Barbeau, Inrap).

Figure 12b: View of the cut of foundation trench 136 in trench 43 (photo S. Barbeau, Inrap). blement final de la structure excavée qui ne peut s'expliquer que par une contemporanéité de l'habitat et de la cave.

La contemporanéité des bâtiments et de la cave (125), suggérée par le plan général des structures, est confirmée par le mobilier céramique.

\section{LA CAVE}

\section{Présentation de la structure}

Le mot " cave " est employé ici en référence à la terminologie définie dans le travail de Stanislas Bossard (2015) sur les Souterrains et autres architectures enterrées de l'âge du Fer en Bretagne et Basse-Normandie. Elle désigne le creusement d'une fosse profonde, dotée d'aménagements en bois, d'accès et d'un plafond artificiel. En Bretagne, la cave boisée est essentiellement reliée à la période des $\mathrm{VI}^{\mathrm{e}}-\mathrm{V}^{\mathrm{e}} \mathrm{s}$.

La cave a été creusée dans les schistes briovériens. Elle se compose d'une fosse-couloir, longue de 14,60 $\mathrm{m}$ et large de 2,40 m maximum en surface (fig. 3 et 16).

La fosse principale a une profondeur comprise entre $1,78 \mathrm{~m}$ au nord et de $1,96 \mathrm{~m}$ au sud. Le fond plat, large de 1,80 $\mathrm{m}$, descend selon une légère pente nord-sud d'un peu plus de $1 \%$, égale à celle du terrain. Les parois sont verticales jusqu'à une hauteur de $1 \mathrm{~m}$ environ puis s'évasent légèrement pour repartir verticalement, sans vraiment former de ressaut (fig. 16), ce qui peut marquer l'emplacement de solives.

L'accès se faisait depuis l'intérieur du bâtiment, à l'extrémité nord-est de la cave où la largeur se réduit à 1,70 m par un système d'échelles encastrées dans la paroi. Structurellement, il subsiste deux encoches, d'environ $0,40 \mathrm{~m}$ de large, visibles dans la partie haute de la paroi nord à $0,22 \mathrm{~m}$ d'écart.

À l'extrémité sud, perpendiculairement à la cave, un creusement rectangulaire équivalent à un petit couloir de $1 \mathrm{~m}$ de large et long de 2,60 m, d'une hauteur maximum de 1,10 m sous le décapage descend en pente douce dans la structure excavée. Il constitue un deuxième accès, utilisé pour acheminer et extraire des produits de la cave (fig. 16).

La cave possède également une niche (186) quadrangulaire de 1,80 m de long par $0,70 \mathrm{~m}$ de profondeur et $0,90 \mathrm{~m}$ de hauteur, creusée en sape dans la paroi est, proche de l'entrée nord (fig. 16).

Des tranchées étroites (entre $0,18 \mathrm{~m}$ et $0,30 \mathrm{~m}$ de large pour une profondeur moyenne de $0,18 \mathrm{~m}$ ) creusées le long de la paroi, au fond de la cave, indiquent l'emplacement de sablières. Elles sont associées à six tranchées transversales qui divisent la cave en 6 modules de taille variable. Le plus long, au nord-est, à l'entrée de la cave a une longueur de $4 \mathrm{~m}$ 

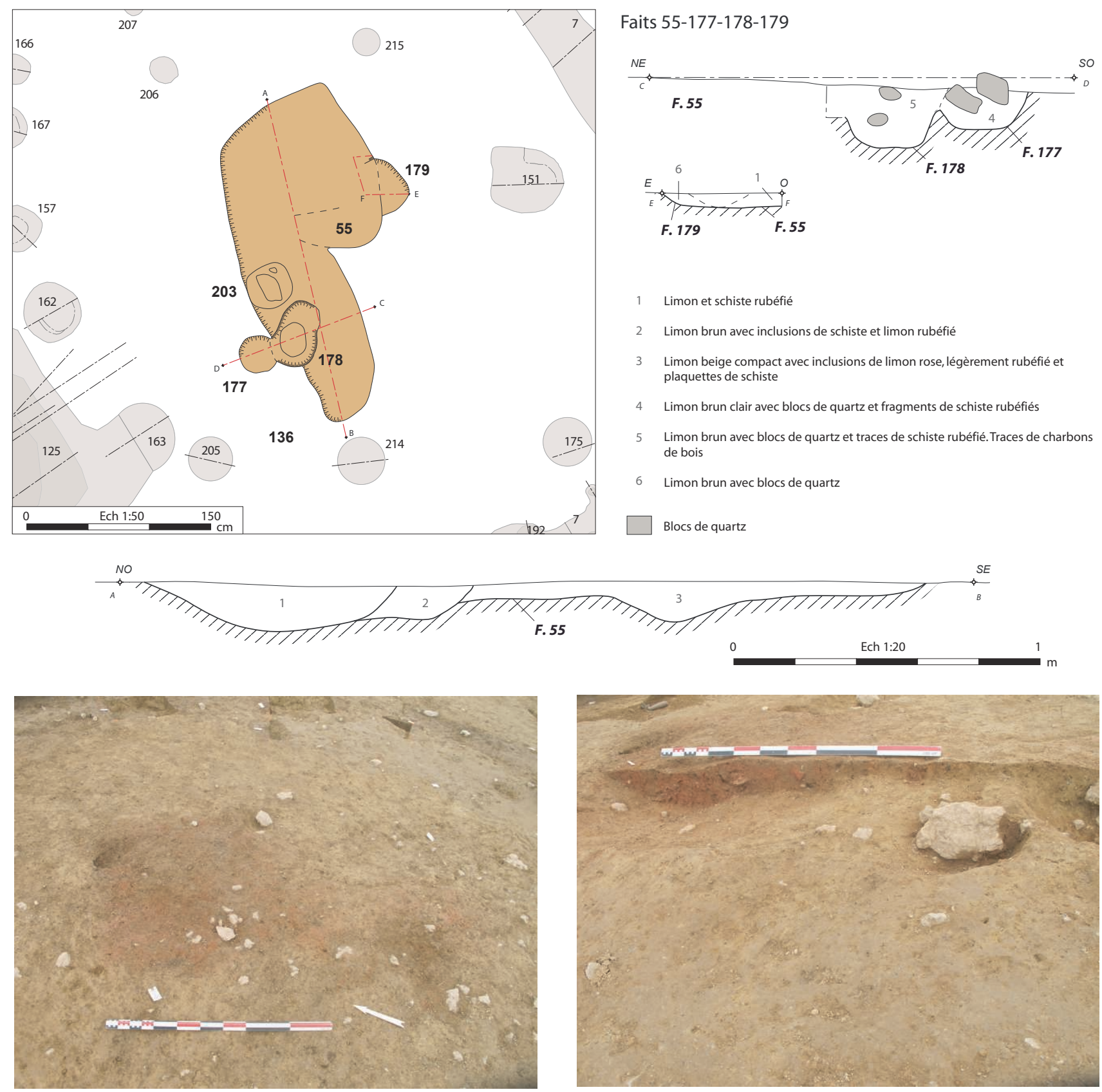

Figure 13: Vue en plan et en coupe du foyer 55 (cliché S. Le Forestier, Inrap).

Figure 13: View in plan and in cut of hearth 55 (photo S. Le Forestier, Inrap).

tandis que le module, à l'extrémité sud, se réduit à 1,30 m de long. Il pourrait exister ici des partitions fermées de portes telles que dans la cave de Kervouyec à Quimper (Finistère) (Nicolas, 2013), avec laquelle elle partage par ailleurs de fortes similitudes. Cette dernière possède trois salles dont les séparations sont marquées au sol par de longues fosses de calage.

L'étude des coupes stratigraphiques révèle un épisode d'effondrement de la partie haute des parois au niveau de la niche à l'entrée nord et la reconstruction du boisage de la paroi à l'avant de l'effondrement dans le prolongement du reste du creusement de la cave marquée par l'installation d'une deuxième tranchée au sol.

Des planches venaient en recouvrement des parois. Elles pouvaient être disposées horizontalement, sur des supports verticaux fixés sur les sablières installées dans les tranchées, ou verticalement, sur un lattis horizontal fixé lui-même sur des montants verticaux assemblés sur les poutres au sol. 


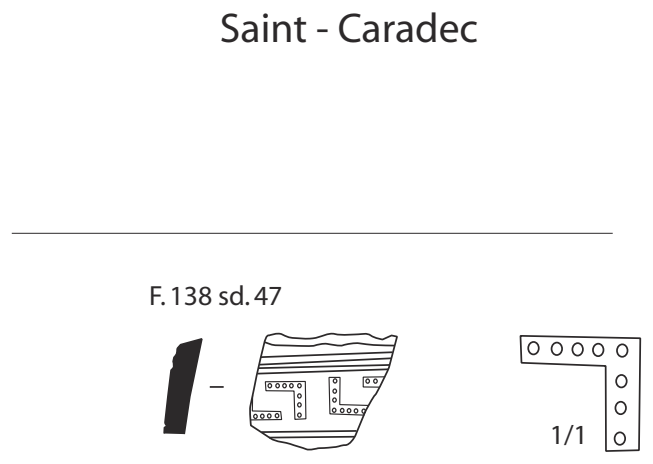

F. 157 us 1

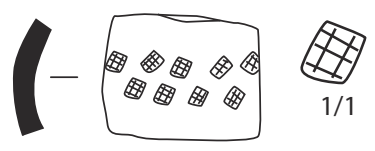

01

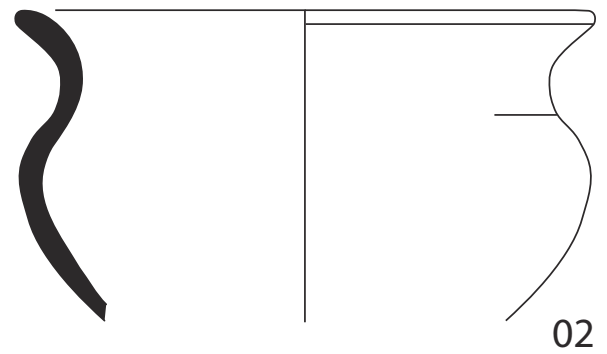

F. 162 sd 63 us 1

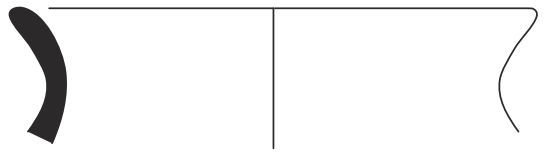

01

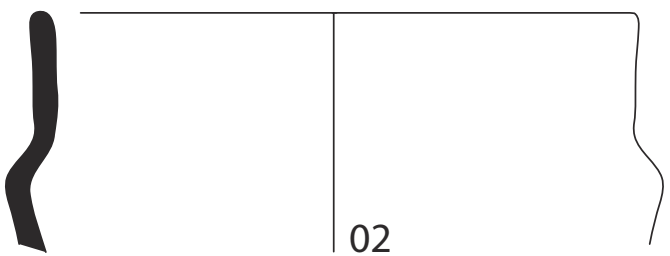

F. 146 , sd 37 us 1

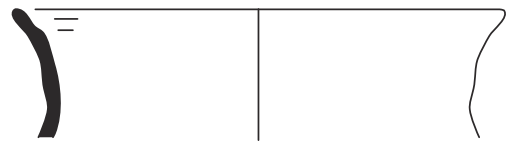

F. 163 sd 62 us 2

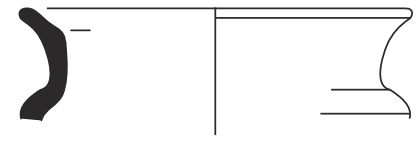

F. 170

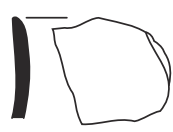

F. 189 (sud) entre 46 et 49

F. 189 (sud) entre 46 et 49
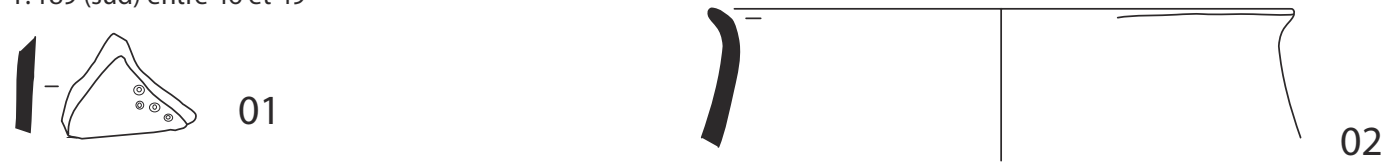

F. 189 proche $s d 46$ associé à TP 84

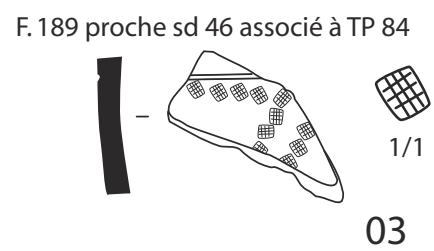

Ech.: $1 / 3$
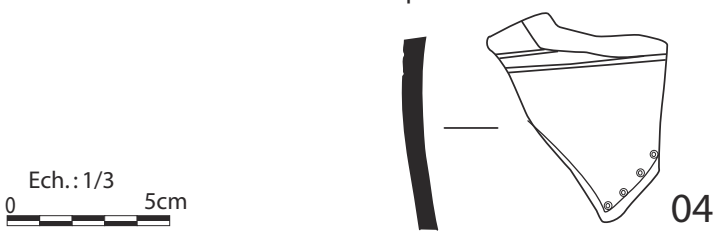

Figure 14 : Planche des fragments de céramique découverts dans les remplissages des tranchées de fondation et les fosses d'implantation de poteau à l'intérieur des bâtiments (DAO H. Delnef, Inrap).

Figure 14: Pottery fragments from the fill of the circular ditches and the post holes inside the building (CAD H. Delnef, Inrap). 
Figure 15: Vue aérienne décorrélée des constructions, des poteaux et de l'entrée de la cave en adéquation avec le plan topographique (DAO E. Collado, Inrap; cliché H. Paitier, Inrap).

Figure 15: Aerial View of the constructions, the postholes and the entrance of the cellar, using the decorrelation technic (CAD E. Collado, Inrap; photo H. Paitier, Inrap).

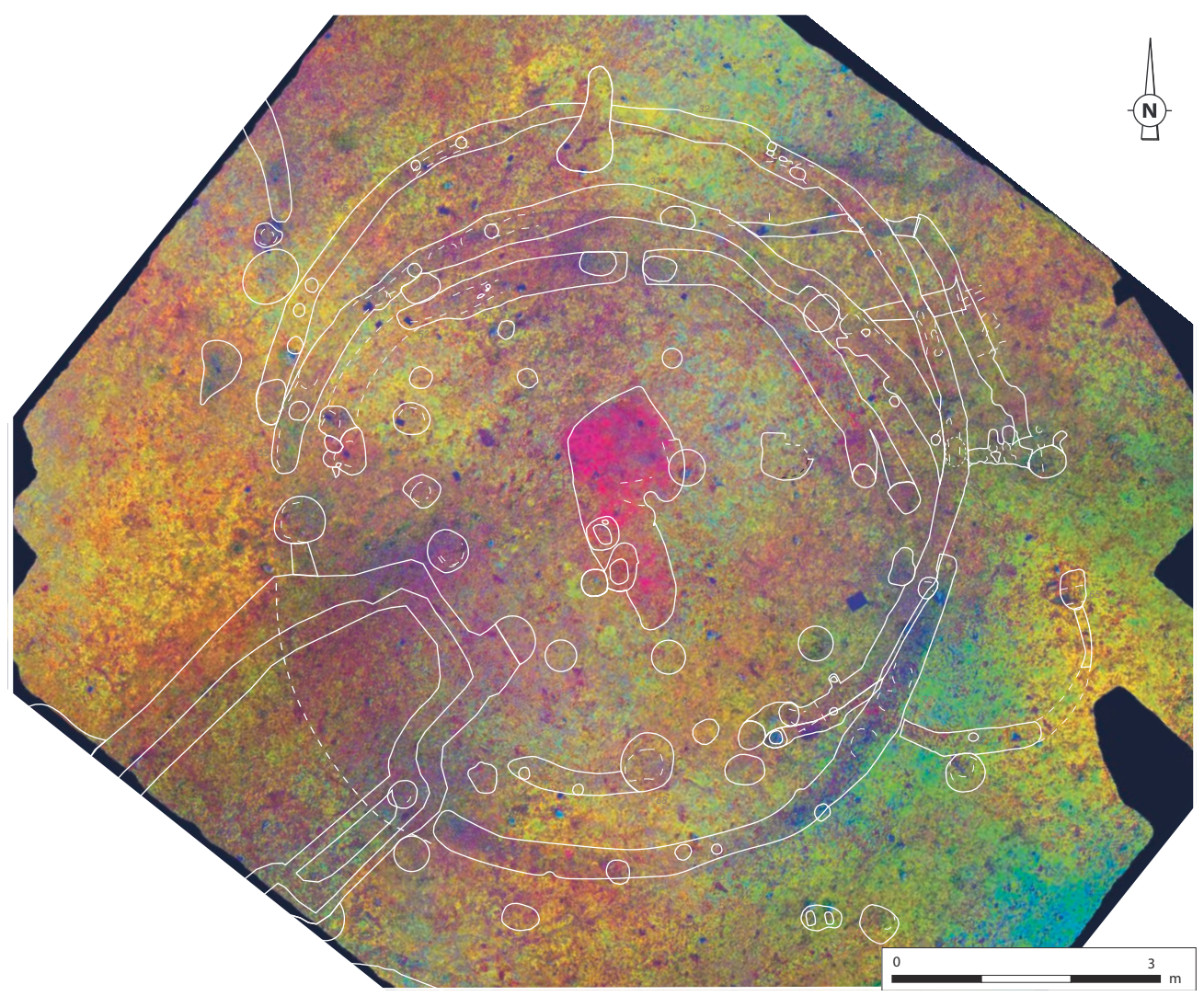

L'espace entre ces planches et la paroi rocheuse, d'une largeur comprise entre $0,10 \mathrm{~m}$ et $0,30 \mathrm{~m}$, est comblé par une succession de couches superposées, alternant une forte épaisseur de limon très argileux jaune orangé et des couches plus fines de plaquettes de schiste gris d'une dizaine de centimètres d'épaisseur (fig. 17). Cette alternance pourrait être d'origine naturelle mais l'hypothèse d'une sélection de ces matériaux et de leur agencement est plausible. La superposition de ces couches pourrait correspondre à un ajout pour rectifier la verticalité de la paroi préalablement à l'installation du boisage, ou encore servir de dispositif d'étanchéification de la cave, la protégeant ainsi des infiltrations d'eau, les niveaux de schiste servent peut-être à absorber les mouvements des limons argileux selon les saisons.

Il n'y a pas de trace extérieure de dispositif de couverture. La cave devait être fermée par un toit de bois recouvert de terre. Le boisage de la cave et le toit de bois et de terre supérieur devaient permettre de supporter la paroi de la maison au-dessus de l'excavation.

Le remblaiement de la cave a été volontairement effectué avant l'abandon du site avec des matériaux essentiellement identiques au substrat extrait de l'excavation, supposant leur stockage à proximité. Le comblement différencié de la partie nord, interne aux bâtiments et composé de gros blocs de quartz et de limons dans les niveaux supérieurs, marque l'abandon définitif de la structure, mais cette différence dans le remplissage pourrait indiquer soit une réutilisation de l'extrémité nord de la cave pour une fonction particulière (telle qu'un cellier, par exemple) pendant un temps, soit un remplissage plus commode et plus rapide depuis l'intérieur de la maison par l'apport de matériaux plus gros (fig. 18 et 19).

\section{Étude du mobilier céramique (par Hélène Delnef)}

Le comblement de la cave a révélé un corpus céramique notable. En effet, au moins douze individus ont au minimum été décomptés. Ainsi, le vase bas 01 monté dans une pâte fine présente un bord évasé avec un léger méplat interne, une courte épaule et une panse carénée. Il appartient à une forme courante entre le début du VI ${ }^{\mathrm{e}}$ et le début du v ${ }^{e}$ s., comme dans l'enclos de Corps-Nuds Launay-Bien (Hamon, 2004) ou à Plérin, dans l'état 1 du fossé d'enclos (Le Gall, 2013; fig. 37, F. 2007/sd 3023/us 6) daté entre la fin $d u v^{e}$ et le début du $v^{e} s$. Le deuxième récipient est une jatte à crans ou encoches en céramique commune telle qu'il est possible d'en voir, par exemple, dans la première phase de Plouër-sur-Rance (Menez, 1996; 237/135-6). Le vase 03 à large bord évasé et fond soulevé trouve un parallèle avec un récipient de Plérin Rue de l'Arrivée (Le Gall, 2013; fig. 68, F. 1016, sd 3015, us 3). 


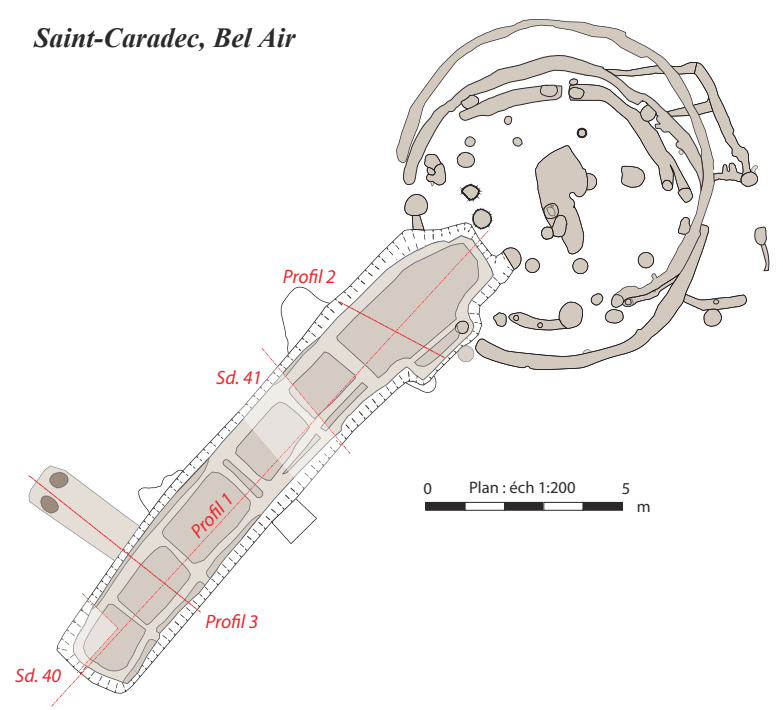

Fait 125

Profil 2 - transversal

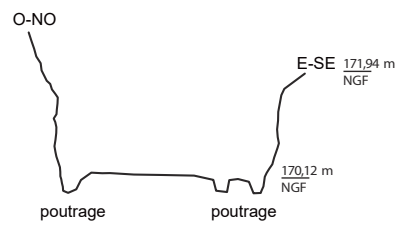

Profil 3 - transversal

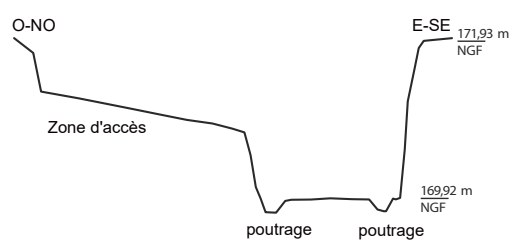

Fait 125 Sd.40
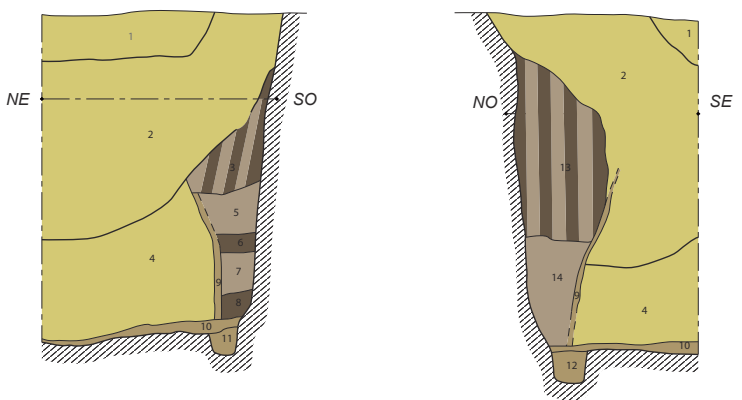

Fait 125

Sd. 41

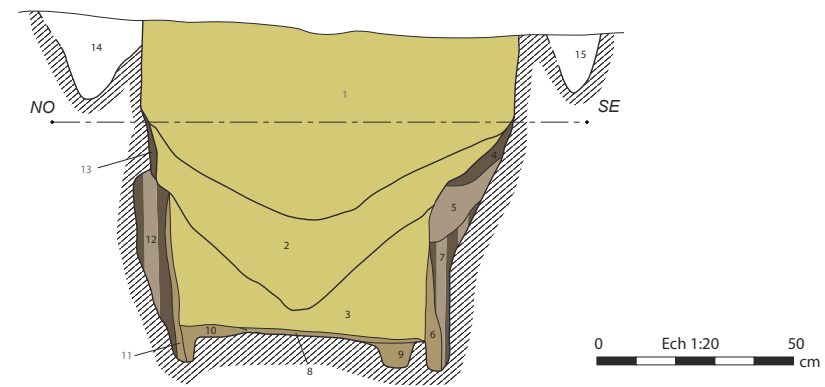

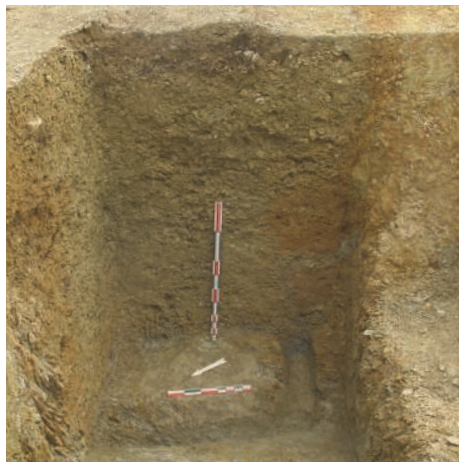

Limon argileux gris bleuté hydromorphe (boisage décomposé)

Limon argileux brun orange

Graviers schisteux gris

Comblement schisteux gris avec de nombreuses plaquettes mêlées à du limon

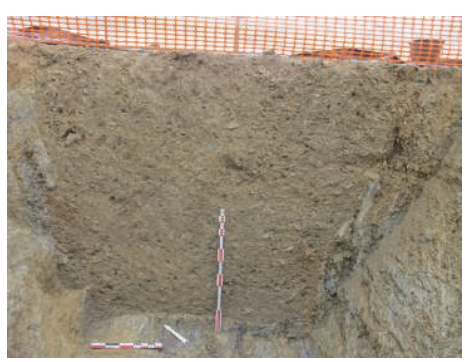

Figure 16 : Profils et coupes de la cave (topographie F. Mélec, Inrap; mise au net A. Mahé, Inrap; DAO E. Collado, Inrap; cliché S. Barbeau, Inrap).

Figure 16: Profiles and cuts of the cellar (survey F. Mélec, Inrap; draft A. Mahé, Inrap; CAD E. Collado, Inrap; photo S. Barbeau, Inrap). 


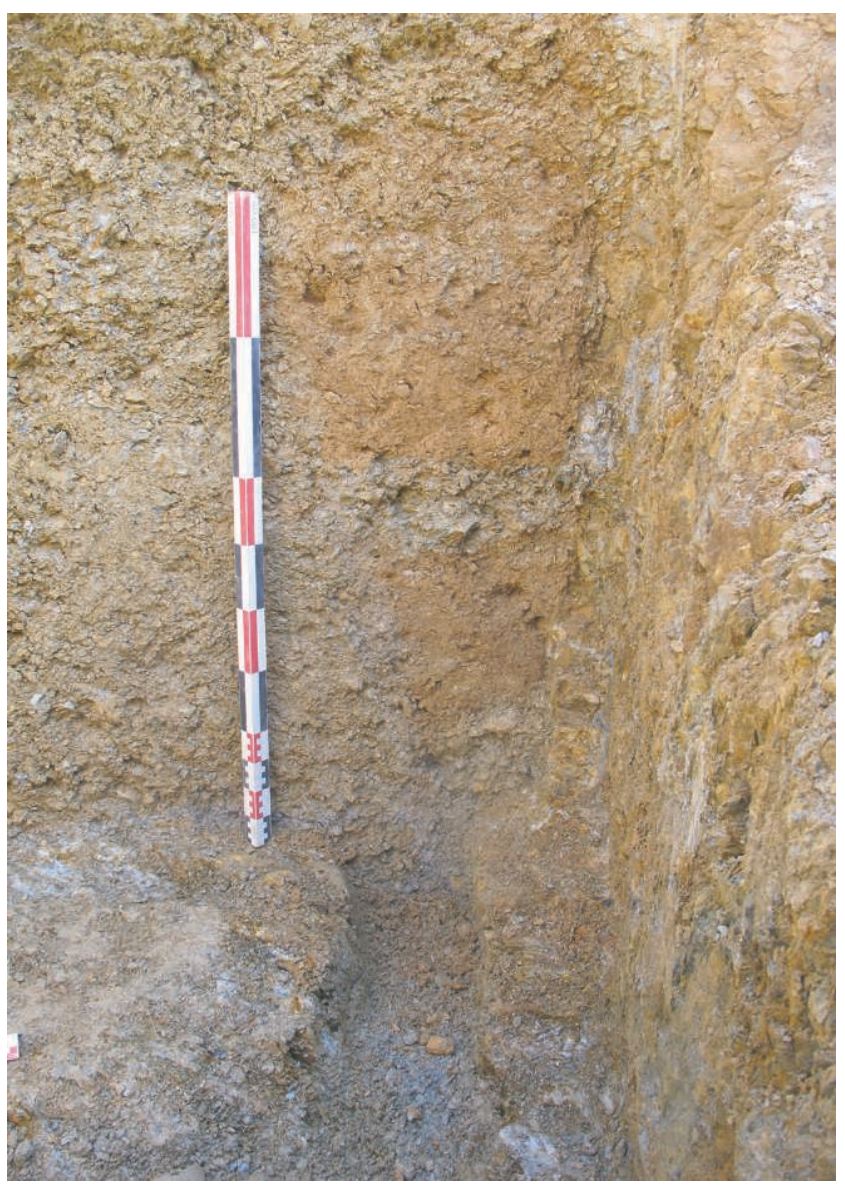

Figure 17 : Détail de l'alternance des couches argileuses et schisteuses entre les planches et la paroi de la cave (cliché : S. Barbeau, Inrap).

Figure 17: Detail of the alternance of clayey and schiste rich fills between the planks and the sides of the cellar (photo S. Barbeau, Inrap).

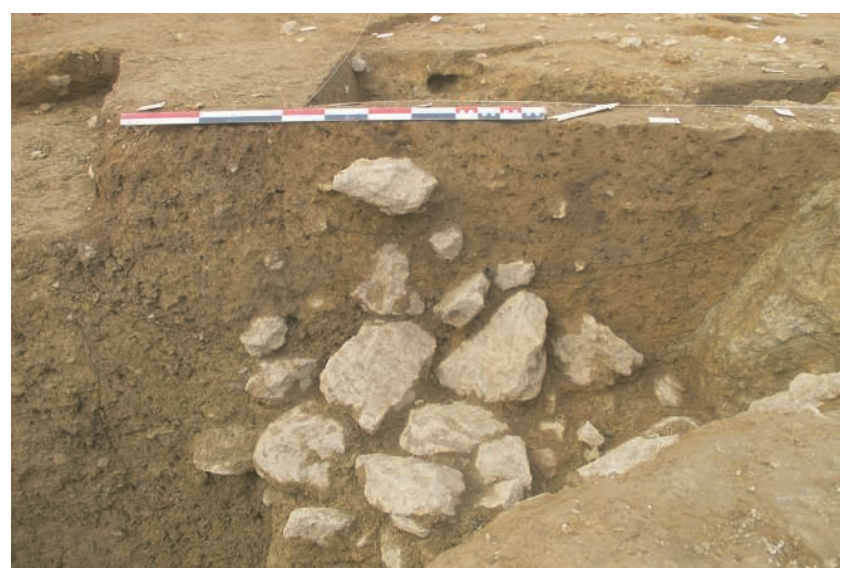

Figure 18 : Vue de la coupe du remplissage de l'entrée (cliché V. Chaigne, Inrap).

Figure 18: View of the cut of the fill of the entrance (photo V. Chaigne, Inrap).
Parmi les formes hautes, le vase 04 à profil caréné et l'individu 05, à courte épaule et de plus grand module, peuvent être comparés à des types courants dans les habitats du Hallstatt final, comme à Quimper Kervouyec II dans le fossé 3720 , daté de la fin du $\mathrm{VI}^{\mathrm{e}}$ s. par une analyse ${ }^{14} \mathrm{C}$ réalisée sur un résidu charbonneux prélevé sur un récipient (Nicolas et al., 2013). Un sixième récipient, découvert dans le fait 125 (coupe B, us 10), est notable, même s'il n'a pu être reconstitué. Il possède un bord digité et un fond plat.

Dans une encoche située à l'extrémité nord de la cave (163), un récipient en céramique fine à bord évasé montrant une légère gorge interne, un col évasé et une courte épaule arrondie est recensé. Celui-ci est proche d'exemplaires découverts à Plérin dans l'état 1 et 2 de l'enclos 2007 (Le Gall, 2013; 63, type B15a), soit entre la fin du vi et le début du v $v^{\mathrm{e}}$.

Le spectre typologique issu de la cave semble légèrement différent de celui des bâtiments par la qualité des individus conservés. Ainsi, les céramiques communes attribuées généralement à des usages utilitaires comme le stockage à court ou moyen terme apparaissent relativement plus nombreuses. La jatte à crans, dont la morphologie est proche des jattes à bord festonné du nord et de l'est de la France, pourrait, de même, servir de support d'éclairage (Malrain et Pinard, 2006, p. 177). On note également l'absence de vase à décor estampé dans ce corpus, contrairement à ce qui est recensé dans les faits 157, 189 et 138 (fig. 14). Le mobilier de la cave concernerait donc des activités domestiques autres que celles liées au service de table, auquel les céramiques estampées du bâtiment pourraient appartenir.

L'ensemble céramique du site de Saint-Caradec se distingue donc par son homogénéité typochronologique. Il trouve de nombreux rapports avec le corpus de Plérin Rue de l'Arrivée et celui de Plouër-sur-Rance. La similarité des motifs estampés en losange quadrillé avec ceux de Guipry est également à souligner. Il complète ainsi nos connaissances de la céramique d'habitat des VI-V $\mathrm{V}^{\mathrm{e}}$ s. en Centre Bretagne.

\section{Hypothèses sur la fonction de la cave}

L'excavation correspond globalement à un volume d'environ $30 \mathrm{~m}^{3}$. Si la fonction première d'une cave est de permettre de stocker et de mettre à l'abri des liquides et/ou des denrées périssables (animales ou végétales), ce que confirme la typologie de la céramique découverte dans les niveaux de remblais, il est plus difficile d'expliquer la segmentation de cette longue cave en 6 modules. Est-elle structurelle ou fonctionnelle?

Structurellement, les tranchées transversales qui séparent les différents espaces pourraient participer au renforcement de la structure excavée et permettre de supporter le poids 


\section{Fait 125}

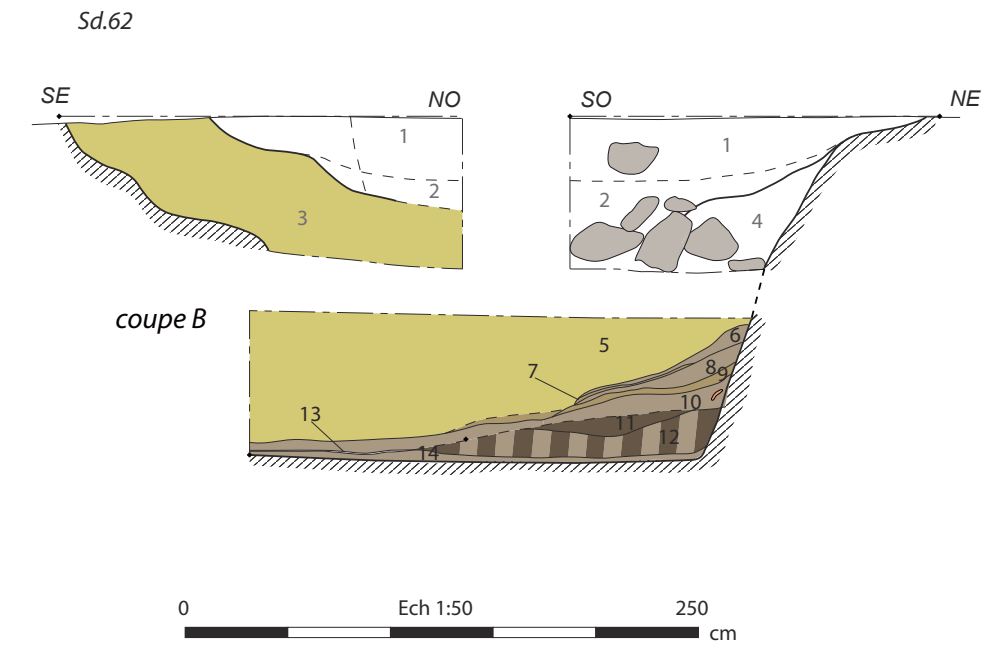

Sd.63

coupe $B$

Figure 19 : Coupes de l'entrée de la cave (DAO E. Collado, Inrap). Figure 19: Cuts of the entrance of the cellar (CAD E. Collado, Inrap).
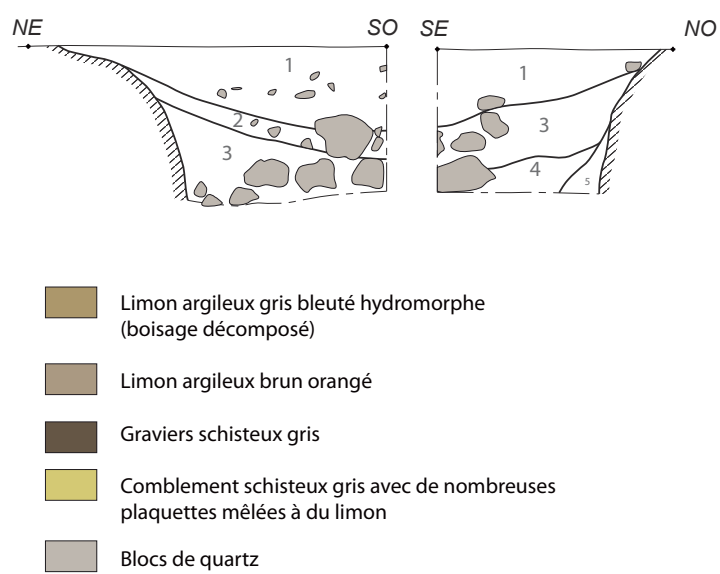

de la toiture et son recouvrement de terre. Elles peuvent également correspondre à la division de la cave en différentes salles cloisonnées.

Des modifications, apportées au cours du temps sur cette excavation (l'adjonction de modules puis au contraire la réduction de l'espace utile), ont été mises en évidence lors de la fouille, mais celles-ci traduisent surtout une évolution dans la quantité de provisions stockées ou la diversification de ces dernières. Cette cave, qui permettait un stockage important de denrées (pour une consommation locale ou pour la vente), nécessitait probablement une rationalisation de l'espace qui se traduirait éventuellement par l'aménagement de salles. Fermées par des portes, les salles pouvaient peut-être aider à la gestion des stocks ou encore éviter des contaminations d'un produit à l'autre (entre des fruits par exemple).

\section{Conclusion}

Une quinzaine de sites possédant des bâtiments circulaires est répertoriée dans l'ouest de la France dont 5 en Bretagne (Godard, 2013). Le corpus très restreint pour cette région s'est vu augmenté récemment, en Bretagne, par la découverte de bâtiments circulaires sur tranchées de fondation sur le site de Kermat 3 à Inzinzac-Lochrist (Le Gall, 2017); une liste, non exhaustive, qui s'enrichit au gré des nouvelles découvertes, telles que celle du site de Bignan (Barbeau, fouille 2017, à paraître), où deux bâtiments circulaires sur tranchées de fondation, situés à l'intérieur d'une enceinte circulaire, ont été fouillés.

La variété des architectures existe au sein même de ce type d'habitat, que ce soit la taille (de 4 à $15 \mathrm{~m}$ de diamètre), le plan, incluant ou non un porche d'entrée, comme sur le site de la Tremblaye 1 à Agneaux (Manche) (Giazzon in Godard, 2013), la technique de construction avec simple poteau central ou structures porteuses, comme sur le site de Poses en Seine-Maritime, ou encore sur poteaux ou avec des tranchées de fondation tel que sur le site de Object'Ifs Sud à Ifs dans le Calvados (Besnard-Vautrin, 2011) ou à Poses (Seine-Maritime) (Godard, 2013). Cependant, aucun d'entre eux ne présente de plan similaire à ceux de Bel Air avec un appendice en forme d'agrafe sur le côté.

Les maisons circulaires, rattachées à une tradition architecturale bien connue en Grande-Bretagne, dès la période de l'âge du Bronze, sont identifiées également depuis longtemps en Normandie. Ce modèle architectural perdure durant le premier âge du Fer jusqu'à la transition avec le second âge du Fer, non seulement sur la façade maritime du quart nordouest de la France mais également dans les régions plus à l'est. Il ne constitue cependant pas l'architecture domestique la plus répandue et fait place peu à peu à une architecture de plan quadrangulaire sur poteau ou tranchée de fondation, une technique majoritairement utilisée durant le second âge du Fer. Le bâtiment circulaire sur tranchées de fondation correspondrait à un modèle intermédiaire qui annonce la généralisation de l'architecture quadrangulaire (Maguer et Le Gall, 2018). 


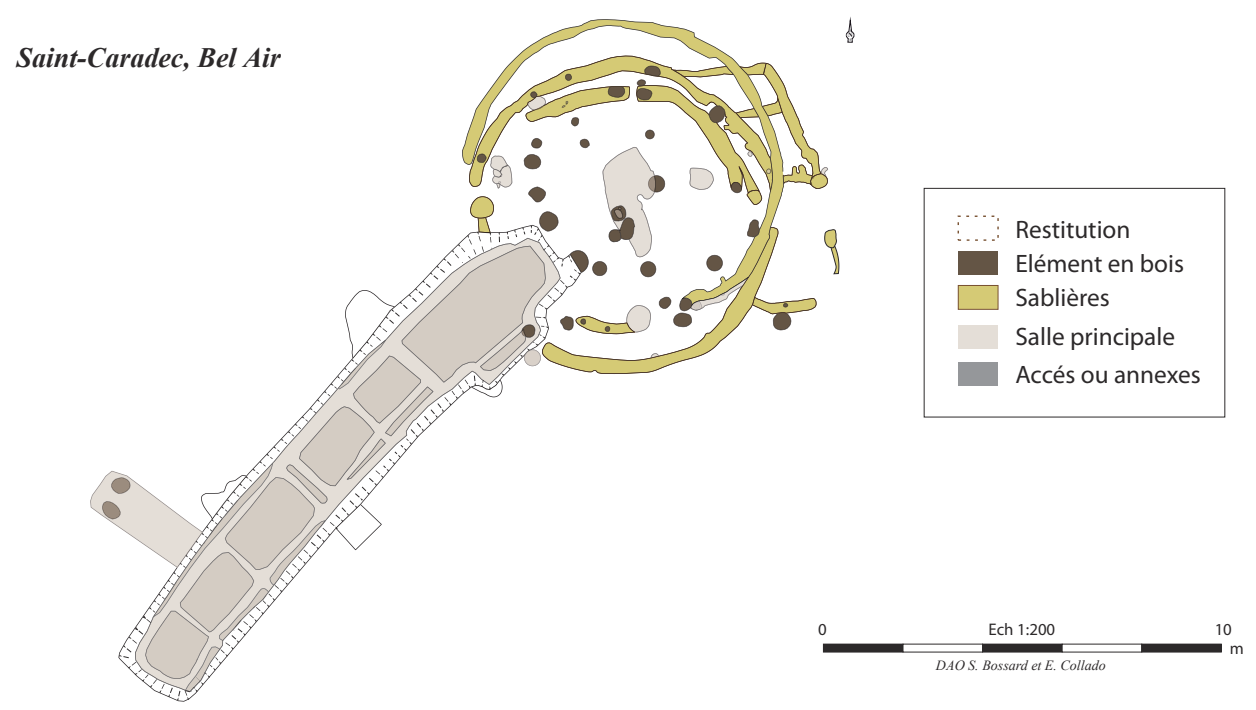

plans des architectures souterraines de type 2.2.

Quimper (29), Kergariou
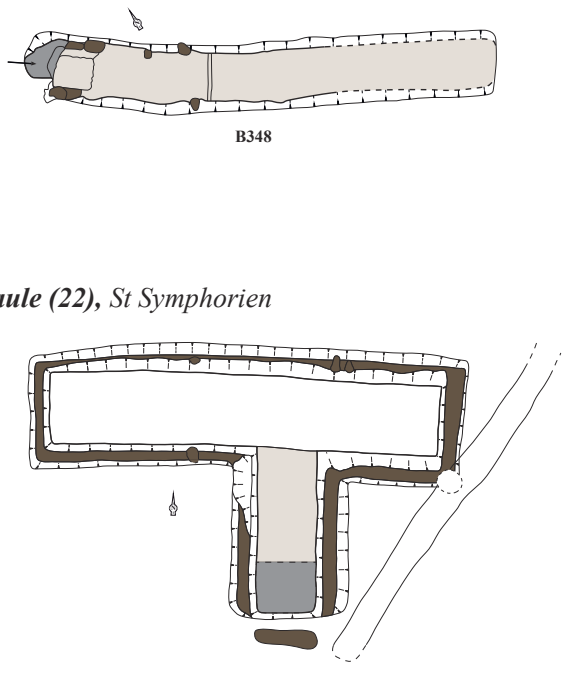

B200

Figure 20 : Comparaison de plans de caves de Bretagne et de Normandie (DAO E. Collado, Inrap, d'après Bossard, 2015). Figure 20: Comparison of plans of the underground passageways of Brittany and Normandy (CAD E. Collado, Inrap, after Bossard, 2015).

Quimper (29), Kervouyec

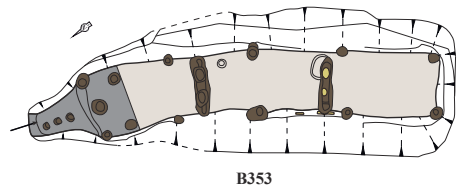

Paule (22), St Symphorien

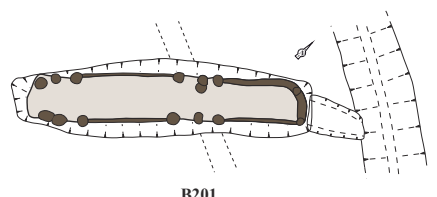

Plans des architectures souterraines de type 2.1.2.

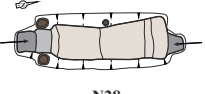

N28
Ifs (14), ZAC Object'Ifs Sud

$$
\text { Agneaux (50), Bellevue }
$$

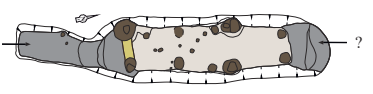

N1
Saint-Martin des Entrées (14), Parc sur l'herbage

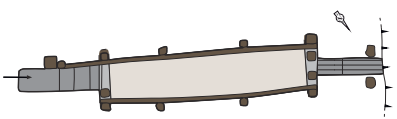

N39
La cave semble avoir été déterminante dans le processus de construction des différents bâtiments au même emplacement, conservant une partie commune au sud-ouest, même si un léger décalage dans les différents plans de construction est notable. Il se pourrait que cette excavation ait connu une phase d'agrandissement (sans qu'il soit possible de l'associer précisément à l'une des phases de construction) avant d'être abandonnée et rebouchée pour une grande partie. Elle sera totalement remblayée avant la fin de l'abandon du site.

La cave de Bel Air s'apparente, par ses dimensions et sa morphologie, à la cave du site de Kervouyec à Quimper datée de la même période. Elle se distingue néanmoins par 
sa technique de boisage sur tranchée et par une niche intérieure (fig. 20).

Les architectures souterraines sont intégrées d'une façon générale à l'habitat, souvent au cœur des établissements de l'âge du Fer. Les emplacements privilégiés sembleraient être les bordures de fossés et le voisinage des habitations (Bossard, 2015, p. 167). Toutefois, si l'association cave-bâtiment de surface est attestée, le lien direct est souvent difficile à établir, chronologiquement et architecturalement. Bien que peu fréquents, ces exemples sont attestés en Bretagne, sur le site de Saint-Symphorien à Paule (Côtes-d'Armor) (Menez, 2009, p. 60) pour la période $\mathrm{du} \mathrm{vI}^{\mathrm{e}}$ au $\mathrm{IV}^{\mathrm{e}}$ s. Le bâtiment d'habitation, beaucoup plus imposant qu'à Bel Air, possède un plan subrectangulaire. La cave se trouve sous le bâtiment et de nombreux souterrains ont également été fouillés sur le site, hors de la maison.

Il existe d'autres exemples de ce lien entre habitation et cave boisée sur le site Route de Kernéost à Bénodet (Finistère) (Roy, 2003), et sur le site de le Boisanne à Plouërsur-Rance (Côtes-d'Armor) (Menez, 1996). Le même constat a été établi en Normandie : sur le site de Bellevue à Agneaux (Marcigny, 2000), le bâtiment d'habitation, de plan rectangulaire sur poteau d'environ $10 \mathrm{~m}$ de long, est manifestement relié à la cave.

Si l'ensemble architectural souterrain de Bel-Air reste globalement semblable à celui des caves répertoriées en Bretagne, le dispositif de tranchées pour le boisage constitue un exemple original qui évoque la technique de construction des bâtiments en surface utilisant la technique de construction sur tranchée de fondation.

Cette petite habitation circulaire sur tranchée, accompagnée de sa cave, forme un bel ensemble architectural unique en Bretagne.

\section{Bibliographie}

Aubry L., Le Puil-Texier M., 2014 - Un enclos funéraire du $1^{\text {er }}$ $2^{\text {nd }}$ âge du Fer, Domaine de la Bizaie, Guipry (Ille-et-Vilaine), rapport final de fouille archéologique préventive, CessonSévigné/Rennes, Inrap Grand Ouest/SRA Bretagne, 154 p.

Ayàn Vila X. M., 2008 - "A round Iron Age : The Circular House in the Hillforts of the Northwestern Iberian peninsula ", Journal of interdisciplinary Celtic Studies, vol. 6: The celts in the Iberian Peninsula [https://www4.uwm.edu/celtic/ ekeltoi/volumes/vol6/index.html], p. 903-1003.

Barbeau S., 2020 - Un site sidérurgique du haut Moyen Âge, Gohvaria, Bignan (Morbihan), rapport final de fouille archéologique préventive, Cesson-Sévigné/Rennes, Inrap Grand Ouest/SRA Bretagne, 2 vol.

Besnard-VAutrin C.-C., 2011 - Habitats et lieux funéraires protohistoriques et vestiges antiques, Ifs - ZAC Object'Ifs Sud dernière tranche (Calvados), vol. 1 : Cercles de l'âge du Bronze, habitats et mobiliers protohistoriques, rapport final de fouille archéologique préventive, Cesson-Sévigné/Rennes, Inrap Grand Ouest/SRA Bretagne, vol. 3, 812 p.

Blanchet S., 2016 - Occupations rurales protohistoriques, Quelisoye, La Bruyère, Larmor-Plage (Morbihan), rapport final de fouille archéologique préventive, Cesson-Sévigné/Rennes, Inrap Grand Ouest/SRA Bretagne, 139 p.

Bossard S., 2015 - Les souterrains et autres architectures enterrées de l'âge du Fer en Bretagne et Basse-Normandie, mémoire de master 2, université de Nantes, $342 \mathrm{p}$.

Cherel A.-F., Lierville O., Menez Y., Villard-Le Tiec A., JeAn S., Lorho T., 2018 - "Les céramiques gauloises en Bretagne. Évolution des formes et des décors entre le $\mathrm{vI}^{\mathrm{e}} \mathrm{s}$. et le $\mathrm{I}^{\mathrm{er}}$ s. av. n. è. ", dans Menez Y. (dir.), Céramiques gauloises d'Armorique : les dessiner, les caractériser, les dater, Rennes, Presses universitaires de Rennes, p. 253-356.

Dechezleprêtre T., Billard C., Blancquaert G., Fournier P., LANGLOIS J.-Y., 1997 - "Les constructions à plan circulaire de Haute-Normandie ", Revue archéologique de l'Ouest, 14, p. 49-56.

Godard C., 2013 - "L'implantation des habitations circulaires en Bretagne et en Normandie à l'âge du Fer : un échange d'hommes et de savoirs ", Revue archéologique de l'Ouest, 30, p. 165-186.

Hamon A.-L., 2004 - Corps Nuds, Launay-Bien (Ille-et-Vilaine) : un enclos funéraire du premier âge $d u$ Fer, rapport final de fouille archéologique préventive, Cesson-Sévigné/Rennes, Inrap Grand Ouest/SRA Bretagne, 40 p.

LeFort A., 2010 - Opération de fouilles archéologiques sur l'estran d'Urville-Nacqueville, rapport final de fouille archéologique préventive, Cesson-Sévigné / Caen, Inrap Grand Ouest / SRADRAC Basse-Normandie, $92 \mathrm{p}$.

Le Gall J., 2013 - Une ferme gauloise du ve s. et des vestiges de l'âge du Bronze, Plérin (Côtes-d'Armor), rapport final de fouille archéologique préventive, Cesson-Sévigné/Rennes, Inrap Grand Ouest/SRA Bretagne, 244 p.

Le Gall J., 2017 - Un grand domaine agricole des $V I^{e}-V^{e}$ s. av. n. è., Kermat III, Inzinzac-Lochrist (Morbihan), rapport final de fouille archéologique préventive, Cesson-Sévigné/Rennes, Inrap Grand Ouest/SRA Bretagne, 1 vol., 368 p.

Maguer P., Le Gall J., 2018 - « Maisons et dépendances dans le nord-ouest de la France au second âge du Fer ", dans VILLARDLe Tiec A. (dir.), Menez Y., Maguer P., Architecture de l'âge $d u$ Fer en Europe occidentale et centrale, actes du $40^{\circ}$ colloque international de l'AFEAF (Rennes, 4-7 mai 2016), Rennes, Presses universitaires de Rennes, p. 303-326.

Malrain F., Pinard E., 2006 - Les sites laténiens de la moyenne vallée de l'Oise du v $v^{e}$ au $I^{e r}$ s. av. n. è. : contribution à l'histoire de la société gauloise, Revue archéologique de Picardie, numéro spécial 23, $268 \mathrm{p}$. 
Marcigny C., 2000 - Agneaux (Manche), Bellevue et la Croix Carrée, rapport de fouille archéologique préventive, Caen, Afan/documentation du SRA Basse-Normandie, p. 40-54.

Menez Y., 1996 - Une ferme de l'Armorique gauloise : Le Boisanne à Plouër-sur-Rance (Côtes-d'Armor), Paris, Éditions de la Maison des sciences de l'homme, 272 p. (Documents d'Archéologie Française, 58).

Menez Y., 2009 - Le Camp de Saint-Symphorien à Paule (Côtesd'Armor) et les résidences de l'aristocratie du second âge du Fer en France septentrionale, mémoire de thèse, université de Paris 1, 2 vol., 1132 p.

Moore T., 2003 - Iron age societies in the severn-Cotswolds. Developing narratives of social and landscape change, thèse non publiée, université de Durham.

Moore T., 2007 - «The Early to later Iron Age transition in the Severn-Cotswolds: enclosing the household? ", dans Pope R., Haselgrove C., The earlier Iron Age in Britain and the near Continent, Oxford, Oxbow Books, p. 259-278.

Nicolas E. (dir.), Le Gall J., Brisotto V., Cherel A.-F., 2013 De multiples occupations protohistoriques, Kervouyec II, Quimper
(Finistère), rapport final de fouille archéologique préventive, Cesson-Sévigné/Rennes, Inrap Grand Ouest/SRA Bretagne, $308 \mathrm{p}$.

Pithon M., 2000 - La Verrie (Vendée), L'Emonnière, Autoroute A 87 Mortagne-sur-Sèvre - La Roche-Sur-Yon, rapport de fouilles archéologiques préventives, Nantes, Afan.

Roy E., 2003 - Découverte d'une entité agricole de l'âge du Fer "Route de Kernéost "à Bénodet (Finistère), rapport final de fouille archéologique préventive, Cesson-Sévigné/Rennes, Inrap Grand Ouest/SRA Bretagne, $64 \mathrm{p}$.

Silva A. C. F., 1986 - A cultura castreja no Noroeste de Portugal, Paços de Ferreira, chambre municipale, musée archéologique de Citânia de Sanfins.

Vaginay M., Guichard V. (dir.), Aulas C., Gentric G., Morel J.-P., VILA E., 1988 - L'habitat gaulois de Feurs (Loire) - Fouilles récentes (1978-1981), Paris, éditions de la Maison des sciences de l'homme, 199 p. (Documents d'Archéologie Française, 14). 